\title{
Axion like particles and the inverse seesaw mechanism
}

\author{
C.D.R. Carvajal, ${ }^{a}$ A.G. Dias, ${ }^{a}$ C.C. Nishi ${ }^{b, c}$ and B.L. Sánchez-Vega ${ }^{d}$ \\ ${ }^{a}$ Universidade Federal do ABC, Centro de Ciências Naturais e Humanas, \\ Av. dos Estados, 5001, 09210-580, Santo André, SP, Brasil \\ ${ }^{b}$ Maryland Center for Fundamental Physics, University of Maryland, \\ College Park, MD 20742, U.S.A. \\ ${ }^{c}$ Universidade Federal do ABC, Centro de Matemática, Computação e Cognição, \\ Av. dos Estados, 5001, 09210-580, Santo André, SP, Brasil \\ ${ }^{d}$ Argonne National Laboratory, \\ 9700 S. Cass Avenue, Argonne, Illinois 60439, U.S.A. \\ E-mail: crisdaruiz@gmail.com, alex.dias@ufabc.edu.br, \\ celso.nishi@ufabc.edu.br, brucesanchez@anl.gov
}

ABSTRACT: Light pseudoscalars known as axion like particles (ALPs) may be behind physical phenomena like the Universe transparency to ultra-energetic photons, the soft $\gamma$-ray excess from the Coma cluster, and the $3.5 \mathrm{keV}$ line. We explore the connection of these particles with the inverse seesaw (ISS) mechanism for neutrino mass generation. We propose a very restrictive setting where the scalar field hosting the ALP is also responsible for generating the ISS mass scales through its vacuum expectation value on gravity induced nonrenormalizable operators. A discrete gauge symmetry protects the theory from the appearance of overly strong gravitational effects and discrete anomaly cancellation imposes strong constraints on the order of the group. The anomalous U(1) symmetry leading to the ALP is an extended lepton number and the protective discrete symmetry can be always chosen as a subgroup of a combination of the lepton number and the baryon number.

Keywords: Beyond Standard Model, Neutrino Physics, Discrete and Finite Symmetries, Anomalies in Field and String Theories

ARXIV EPRINT: 1503.03502 


\section{Contents}

1 Introduction 1

2 ALP and the inverse seesaw mechanism 3

2.1 Symmetries of the model 9

$\begin{array}{ll}2.2 & \text { Model with one ALP } \\ & 13\end{array}$

$\begin{array}{lll}2.3 & \text { Models with two ALPs } & 16\end{array}$

$\begin{array}{lll}2.3 .1 & \text { Model I } & 17\end{array}$

$\begin{array}{lll}2.3 .2 & \text { Model II } & 18\end{array}$

$\begin{array}{llr}3 & \text { Conclusions } & 20\end{array}$

\section{Introduction}

Pseudo Nambu-Goldstone bosons of very low masses, interacting effectively with the electromagnetic field, are generally predicted in Standard Model (SM) extensions containing approximate continuous global symmetries which are spontaneously broken. A distinctive example of this type of particles is the axion, which arises when the strong CP problem is solved through the Peccei-Quinn mechanism [1-3]. Generically, any light pseudo NambuGoldstone boson whose coupling to photons is similar to that of the axion has been denoted as an axion like particle (ALP). Several experiments are in search of the peculiar effect of photon $\leftrightarrow$ ALP oscillations and these searches guide the development of theories containing these particles [4-6].

The symmetry associated to the ALP is usually taken to be spontaneously broken at a very high energy scale, and we assume here that this breakdown occurs through the vacuum expectation value (vev) of a scalar field singlet under the SM symmetry group. The ALP decay constant, which controls the feeble ALP couplings to other SM particles, can be identified to this vev, up to order one coefficients.

In this work we investigate settings where the same scalar singlet hosting the ALP also gives rise to the mass scales involved in the inverse seesaw (ISS) mechanism for neutrino mass generation, a well-known mechanism that does not require too heavy neutral fermions $[7,8]$. There are two scales beyond the SM in the ISS mechanism. One of them is directly related to the lepton number breakdown. In our proposal the usual lepton number is embedded in an anomalous $\mathrm{U}(1)_{X}$ symmetry associated to the ALP. Such a symmetry is an accidental one in the sense that it automatically arises from the imposition of another symmetry considered to be more fundamental. Breaking of the accidental $\mathrm{U}(1)_{X}$ symmetry is expected from gravitational interactions through nonrenormalizable operators, which also generate the ALP mass. 
Therefore, we also deal with the problem of stabilizing both the mass scales in the ISS mechanism and the ALP mass in face of gravitational interactions. Nonrenormalizable operators that would bring too large mass corrections can be avoided by assuming discrete $\mathbb{Z}_{N}$ symmetries, which are remnants of gauge symmetries valid at very high energies [9]. The choices for the $\mathbb{Z}_{N}$ symmetries are greatly reduced by the conditions they need to satisfy in order to be free from anomalies [10-16]. For example, there are only a handful of interesting anomaly free discrete gauge $\mathbb{Z}_{N}$ symmetries for the MSSM [10-13, 17].

Discrete symmetries have already been used for suppressing dangerous operators that prevents the solution of the strong CP problem in different models containing the axion [1827]. Such symmetries are shown to be crucial in multi-ALP models, where very low mass ALPs need effective protection against gravitational interactions [26].

At the same time that the $\mathrm{U}(1)_{X}$ symmetry breaking scale leads to the correct mass scales for the ISS mechanism, we look for models where the ALP coupling with photons and the ALP mass have values that allow the explanation of three hinted astrophysical phenomena: the anomalous Universe transparency for very energetic $\gamma$-ray [28-37], the soft X-ray excess from the Coma cluster [38-42], and the X-ray line at $3.5 \mathrm{keV}$ [43-46]. These phenomena have already motivated the development of general multi-ALP models, containing an axion dark matter candidate whose decay constant is associated to the high energy scale entering in the canonical seesaw mechanism [26].

The first astrophysical hint, the anomalous Universe transparency, follows from observations of active galactic nuclei (AGN) [30-33, 37]. An ALP coupling with the photon and with mass within some specific interval could provide an explanation to this phenomena through $\gamma$-ray $\leftrightarrow$ ALP oscillations in the magnetic fields of AGNs, and in the intergalactic medium [29, 34-36] (see, however, ref. [47] for a recent analysis of the cosmic transparency hint).

Second, the observed excess of soft X-ray coming from the Coma cluster [38] could be explained assuming a cosmic ALP background radiation, originated from the decay of heavy moduli fields and corresponding to a fractional number of extra neutrinos [39, 40]. The cluster magnetic field would make possible the conversion of the ALP into the observed X-rays [41, 42].

At last, the $3.5 \mathrm{keV}$ line has been reported from observations, using the XMM-Newton satellite data, of the stacked spectrum of galaxy clusters and in the Perseus cluster [43], and also in the Andromeda galaxy [44]. A possible interpretation for this could be the two photon decay of a dark matter ALP with mass equal to $7.1 \mathrm{keV}$ [45, 46] (another possibility could be a CP even scalar [48, 49], or a specific majoron [50, 51]). Even if a $7.1 \mathrm{keV}$ dark matter does not correspond to an ALP, it could decay into ALPs that in turn decay into photons [52]. It has to be said that a study with the Chandra data of X-ray of the Milky Way did not show a conclusive evidence for the $3.5 \mathrm{keV}$ line [53], and that other interpretations for the $3.5 \mathrm{keV}$ line in terms of some specific Potassium and Chlorine lines were also suggested [54]. On the other hand it is argued in [55] that the interpretation of dark matter decay as the origin of the $3.5 \mathrm{keV}$ line is consistent with the XMM-Newton dataset of the Milk Way center. Although there is some debate on the origin of the $3.5 \mathrm{keV}$ line signal we shall assume it is due to an ALP decay. 
We also motivate our work with the new generation of proposed experiments which are projected to probe some regions of the parameter space of the ALP coupling with photons and its mass. Among these experiments, we can mention: the ALPS-II [56], the helioscope IAXO [57], and the observatories PIXIE [58] and PRISM [59]. Most of the models we propose here are within the prospected search range of these experiments. In figure 2 it is shown the regions in the parameter space to be tested by these experiments, as well as the ones allowing for explanation of the hinted astrophysical phenomena.

The outline of the paper is the following: in section 2 we present the general setting that relates the physics of an ALP, its astrophysical motivations and the generation of the ISS scales. In subsequent section 2.1, we analyze the general symmetry properties of the models and establish necessary conditions for interesting models. Then in sections 2.2 and 2.3 we show, respectively, models with one and two ALPs. Finally, We conclude in section 3 .

\section{ALP and the inverse seesaw mechanism}

We start by showing the main elements that need to be considered in our constructions containing just one complex scalar field whose vev generates the energy scales involved in the ISS mechanism, and which are assumed to be associated with new physics beyond the SM. Such scales are taken as being proportional to a scalar field vev times a suppression factor, composed by this vev divided by the Planck scale and raised to some power. The complex scalar field hosts an ALP which, through its effective interaction with the electromagnetic field, is going to provide explanation for astrophysical phenomena like the soft X-ray excess and the Universe transparency. For this, the ALP needs to have its mass protected from dangerous effective operators due to gravitational interactions. In order to obtain the natural ISS mechanism scales and the appropriate mass for the ALP, we look for suitable discrete symmetries over the fields.

In the ISS mechanism [7,8], two extra sets of neutral fermionic singlet fields, $N_{i R}$ and $S_{i R}, i=1,2,3$, are taken into account in addition to the SM neutrino fields $\nu_{i L}$. It is assumed that after spontaneous symmetry breaking, a mass Lagrangian is generated containing the following terms

$$
-\mathscr{L} \supset \overline{N_{R}} m_{D} \nu_{L}+\overline{S_{R}} M N_{R}^{c}+\frac{1}{2} \overline{S_{R}} \mu S_{R}^{c}+\text { H.c. },
$$

with the $3 \times 3$ Dirac mass matrices $m_{D}, M$, and the Majorana mass matrix $\mu$, which without loss of generality can be taken diagonal. The mass matrix texture arising from eq. (2.1), with the basis choice $\left[\nu_{L}, N_{R}^{c}, S_{R}^{c}\right]$, is

$$
\mathbf{M}=\left[\begin{array}{ccc}
0 & m_{D}^{\top} & 0 \\
m_{D} & 0 & M^{\top} \\
0 & M & \mu
\end{array}\right]
$$

It was observed by Mohapatra and Valle [7, 8] that a mass matrix of the form in eq. (2.2) may lead to three active neutrinos with masses at the sub-eV scale, without invoking very 
large entries in the matrix $\mathbf{M}$. For example, masses at the sub-eV scale for the active neutrinos can be obtained with the entries of $m_{D}, M$, and $\mu$ of order $10 \mathrm{GeV}, 1 \mathrm{TeV}$, and $1 \mathrm{keV}$, respectively. Specifically, the lepton number is only broken by a small scale set by $\mu$, which is the inverse of what is assumed in the canonical seesaw mechanism where lepton number is broken by a very large right-handed neutrino scale. Taking a matrix expansion in powers of $M^{-1}$, block diagonalization of $\mathbf{M}$ leads to the approximate mass matrix for the three light active neutrinos

$$
m_{\nu} \approx m_{D}^{\top} M^{-1} \mu\left(M^{\top}\right)^{-1} m_{D}
$$

and a $6 \times 6$ matrix

$$
M_{R} \approx\left[\begin{array}{cc}
0 & M^{\top} \\
M & \mu
\end{array}\right],
$$

related to six neutrinos. These last ones are supposedly heavier than the active neutrinos, with masses at the scale of $M$, and are quasi-degenerate (pseudo-Dirac nature) if the entries of $\mu$ are small compared to the ones in $M$. If the number of $S_{i R}$ fields were greater than the number of $N_{i R}$ fields, one or more neutrino states with masses at the $\mu$ scale would arise, and they could also contribute as dark matter [60] (another possibility for keV DM in the ISS mechanism is given in ref. [61]). The mixing between the heavy neutrinos and active neutrinos is approximately given by $\epsilon=m_{D} M^{-1}$ and unitarity violation effects are typically of the order $\epsilon^{2}$. General aspects of the ISS mechanism concerning the neutrino mixing and violation of unitarity were developed in refs. [62-66]. Generically, $\epsilon^{2}$ at the percent level is not excluded experimentally, but may be within the reach of future experiments probing lepton flavor violating transitions [64-66] and direct production of heavy states at colliders $[67,68]$.

The scales involved in $M$ and $\mu$ are supposed to arise from new physics beyond the SM. In particular, the $\mu$ term in eq. (2.1) breaks the lepton number symmetry explicitly. From the point of view of naturalness it is reasonable that the nonvanishing entries of $\mu$ be associated with a small effective energy scale compared to the electroweak scale, $v_{\mathrm{w}}=246 \mathrm{GeV}$. In the limit $\mu \rightarrow 0$ lepton number conservation is restored increasing the set of symmetries. Thus, the entries of $\mu$ are expected to be small in comparison with the mass scales of the SM, which contain the lepton number as a global automatic symmetry.

In our approach, the parameters $\mu$ and $M$ are gravity induced and result from the very high vev of the complex scalar field times suppression factors containing the Planck scale, with the parameters in $m_{D}$ proportional to $v_{\mathrm{w}}$. This contrast with proposals where the typical energy scale in $M$ is due to a new theory with spontaneous symmetry breaking at the TeV scale [69-71].

The complex scalar field is a singlet under the SM gauge group and has a vev denoted as $\langle\sigma\rangle=v_{\sigma} / \sqrt{2}$, with

$$
10^{9} \mathrm{GeV} \lesssim v_{\sigma} \lesssim 10^{14} \mathrm{GeV},
$$

defining the intermediate scale range. This leads to a photon-ALP coupling with value required to explain astrophysical phenomena, with the ALP detectable by future experi- 
ments $[26,72]$. We parameterize the scalar singlet as

$$
\sigma(x)=\frac{1}{\sqrt{2}}\left[v_{\sigma}+\rho(x)\right] e^{i \frac{a(x)}{v_{\sigma}}},
$$

were $a(x)$ is the ALP field. The radial field $\rho(x)$ gets a mass at the scale $v_{\sigma}$ and we assume it decouples from the low energy effective theory. In the models presented here $\sigma$ carries charge of a global $\mathrm{U}(1)_{X}$ chiral symmetry which is explicitly broken by the gravitational interactions in such a way that, after spontaneous symmetry breaking, the ALP gets a small mass. The $\mathrm{U}(1)_{X}$ symmetry is taken as accidental meaning that it results from one or more imposed gauge discrete $\mathbb{Z}_{N}$ symmetries — not broken by gravitational interactions - restricting the main interactions of the neutral fermion fields with the scalar fields being

$$
\mathscr{L} \supset \overline{N_{R}} y \widetilde{H}^{\dagger} L+\frac{\sigma^{p}}{M_{\mathrm{Pl}}^{p-1}} \overline{S_{R}} \eta N_{R}^{c}+\frac{1}{2} \frac{\sigma^{q}}{M_{\mathrm{Pl}}^{q-1}} \overline{S_{R}} \zeta S_{R}^{c}+\text { H.c. }
$$

where $y, \eta$ are complex $3 \times 3$ matrices, and $\zeta$ is a symmetric $3 \times 3$ matrix. $L_{i}$ and $H$ are the leptons and Higgs $\mathrm{SU}(2)_{L}$ doublet fields, respectively, with $\widetilde{H}=i \tau_{2} H^{*}$. The complex conjugate field $\sigma^{*}$ can be equally considered in the third term, instead of $\sigma$, while we conventionally define the scalar present in the second term to be $\sigma$. We use the reduced Planck scale $M_{\mathrm{Pl}}=2.4 \times 10^{18} \mathrm{GeV}$ for the gravitational scale. The vev of the Higgs doublet field is $\langle H\rangle=\left(0, v_{\mathrm{w}} / \sqrt{2}\right)^{T}$. We will see that $\mathrm{U}(1)_{X}$ is directly related to an extended lepton number and thus, in our approach, the smallness of $\mu$ follows from its explicit but small breaking due to gravity $\left(1 / M_{\mathrm{Pl}}\right.$ suppression) and its spontaneous breaking at the scale $v_{\sigma}$.

With the vev of the scalar fields in eq. (2.7), the effective Lagrangian in eq. (2.1) is obtained with the mass matrices

$$
m_{D}=y \frac{v_{\mathrm{w}}}{\sqrt{2}}, \quad M=\eta \frac{v_{\sigma}^{p}}{2^{\frac{p}{2}} M_{\mathrm{Pl}}^{p-1}}, \quad \mu=\zeta \frac{v_{\sigma}^{q}}{2^{\frac{q}{2}} M_{\mathrm{Pl}}^{q-1}} .
$$

$m_{D}$ is naturally at the $100 \mathrm{GeV}$ scale without requiring the entries of $y$ to be fine tuned. The nonvanishing entries of $\eta$ and $\zeta$ are all of order one, under the assumption that the nonrenormalizable interactions in eq. (2.7) are exclusively due to gravitational interactions, whose universal coupling is $1 / M_{\mathrm{Pl}}$. Thus, we look for values of $v_{\sigma}$ in which the mass scale function $F(k)=v_{\sigma}^{k} / 2^{\frac{k}{2}} M_{\mathrm{Pl}}^{k-1}$ is assumed to have values $F(p)=0.1-10 \mathrm{TeV}$ and $F(q)=0.1-10 \mathrm{keV}$, for $p$ and $q$ integers. In figure 1, curves for $p$ and $q$ are shown as functions of $v_{\sigma}$. Within the range in eq. (2.5), we can see that only

$$
p=2,3 \text { and } q=3,4,5,
$$

can generate the appropriate scales for the ISS mechanism. Moreover, if only one vev accounts for both $M$ and $\mu$, it can be seen that there are only two sets of solutions:

$$
\begin{array}{ll}
(p, q)=(2,3) & \text { for } \quad v_{\sigma} \approx 2.2 \times 10^{10} \mathrm{GeV}-5.5 \times 10^{10} \mathrm{GeV} \\
(p, q)=(3,5) & \text { for } \quad v_{\sigma} \approx 2.8 \times 10^{13} \mathrm{GeV}-5.5 \times 10^{13} \mathrm{GeV}
\end{array}
$$

However, we should keep in mind that the scale for $\mu$ is more flexible than $M$. 


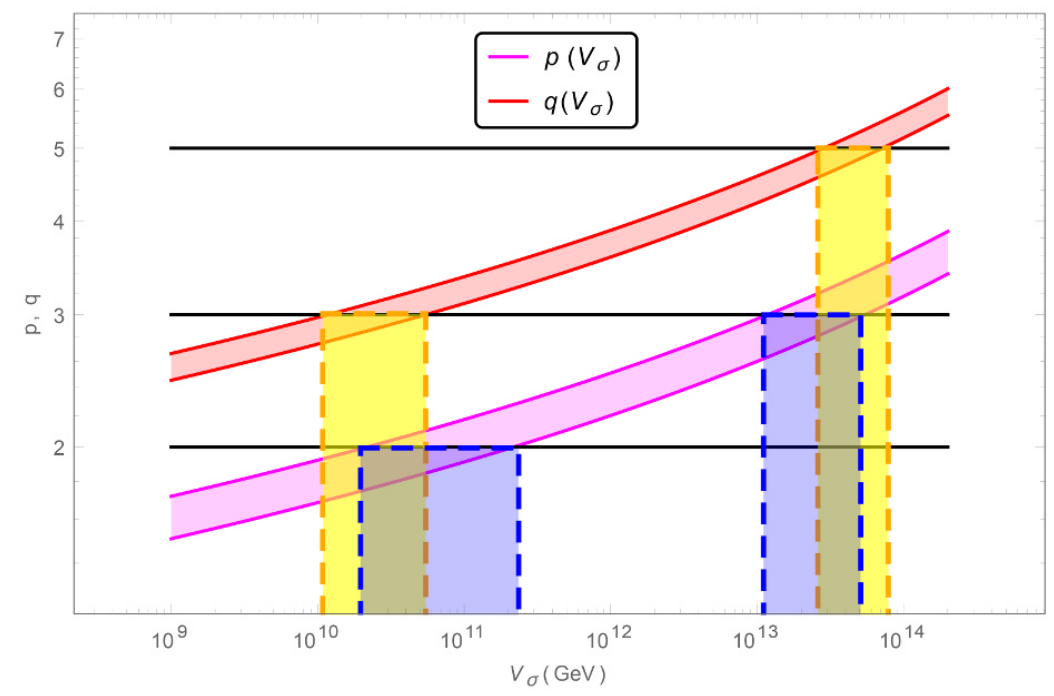

Figure 1. $p$ (purple) and $q$ (red) as a function of $v_{\sigma}$ for $F(p)=0.1-10 \mathrm{TeV}$ and $F(q)=0.1-10 \mathrm{keV}$, with $F(k)=v_{\sigma}^{k} / 2^{\frac{k}{2}} M_{\mathrm{Pl}}^{k-1}$. The regions of intersection between yellow and blue bands indicate common values for $v_{\sigma}$, possible for the set of integers $(p, q)=(2,3)$ or $(p, q)=(3,5)$.

We observe that for both sets of values of $(p, q)$ in eq. (2.10) the active neutrinos masses are independent of the Planck mass, at leading order, and it happens whenever $q=2 p-1$. This feature would not be possible if, e.g., neutrinos masses were generated by type-I seesaw with heavy masses induced by gravity. In fact, eq. (2.3) leads, in face of eq. (2.8), to

$$
m_{\nu} \approx y^{\top} \eta^{-1} \zeta\left(\eta^{\top}\right)^{-1} y \frac{v_{\mathrm{w}}^{2}}{\sqrt{2} v_{\sigma}}
$$

This formula shows that the active neutrinos masses have a suppression factor $v_{\mathrm{w}} / v_{\sigma}$ in relation to the electroweak scale, $v_{\mathrm{w}}$. Such an explanation for having small neutrinos masses resembles the canonical seesaw mechanism and have been observed in other models implementing the ISS mechanism [69].

Now we show that besides having its value constrained to be within a range that allows active neutrinos to have masses at the sub-eV scale, $v_{\sigma}$ can also furnish an ALP-photon coupling as required for explaining the mentioned astrophysical phenomena. It is supposed here that such ALP-photon coupling arises effectively by means of the $\sigma$ field interaction with a new colorless fermion, $E$, which carries one unit of electric charge and is a singlet under $\mathrm{SU}(2)_{L}$ group. Along with $\sigma, N_{i R}$, and $S_{i R}$, we assume that $E$ also carries charge of $\mathrm{U}(1)_{X}$. Under this symmetry these fields transform as

$$
\begin{aligned}
\sigma & \rightarrow e^{i \beta} \sigma, & \\
N_{i R} & \rightarrow e^{i X_{N} \beta} N_{i R}, & S_{i R} \rightarrow e^{i X_{S} \beta} S_{R i}, \\
E_{L} & \rightarrow e^{i X_{E_{L}} \beta} E_{L}, & E_{R} \rightarrow e^{i X_{E_{R}} \beta} E_{R},
\end{aligned}
$$

with charges $X_{\psi}$, normalized such that $X_{\sigma}=1$. 
The effective Lagrangian describing the interactions of the ALP with photons is

$$
\mathscr{L}_{a \gamma}=\frac{1}{2} \partial_{\mu} a \partial^{\mu} a-\frac{1}{2} m_{a}^{2} a^{2}-\frac{g_{a \gamma}}{4} a F_{\mu \nu} \tilde{F}^{\mu \nu},
$$

where $F_{\mu \nu}$ is the electromagnetic field strength and $\tilde{F}^{\mu \nu}=\epsilon^{\mu \nu \lambda \rho} F_{\lambda \rho} / 2$ its dual. The ALPphoton coupling constant, $g_{a \gamma}$, is given by

$$
g_{a \gamma}=\frac{\alpha}{2 \pi} \frac{C_{a \gamma}}{v_{\sigma}}
$$

where $\alpha \approx 1 / 137$, and the anomaly coefficient $C_{a \gamma}$ depends on the $\mathrm{U}(1)_{X}$ and electric charges of the fermionic fields $\psi_{i}, X_{\psi_{L, R}}$ and $C_{\mathrm{em}}^{(i)}$, respectively, according to

$$
C_{a \gamma}=2 \sum_{\psi}\left(X_{\psi_{L}}-X_{\psi_{R}}\right)\left(C_{\mathrm{em}}^{(\psi)}\right)^{2}
$$

Throughout this work only the field $E$ is chiral under $\mathrm{U}(1)_{X}$ - the left- and right-handed components of $E$ have different $\mathrm{U}(1)_{X}$ charges - so that eq. (2.15) reduces to $C_{a \gamma}=$ $2\left(X_{E_{L}}-X_{E_{R}}\right)$. The effective coupling in eq. (2.14) can be obtained through a rotation of the fermionic fields $\psi \rightarrow e^{i X_{\psi} \frac{a(x)}{v_{\sigma}}} \psi$ which does not leave the integration measure invariant, meaning that the $\mathrm{U}(1)_{X}$ symmetry is anomalous (for details see ref. [73]). With such a rotation it turns out that the ALP has only derivative couplings with the fermions. We omit the interactions of the ALP with fermions since these effects are outside the scope of this work.

A nonzero value for $m_{a}$ in eq. (2.13) must be generated by an explicit breaking of $\mathrm{U}(1)_{X}$, characterizing the ALP as a pseudo Nambu-Goldstone boson. We follow the assumption that gravitational interactions do not respect any global continuous symmetry and that $\mathrm{U}(1)_{X}$ is explicitly broken by nonrenormalizable operators suppressed by $M_{\mathrm{Pl}}$. However, as argued by Krauss and Wilczek [9], discrete symmetries like $\mathbb{Z}_{N}$, which are leftover of gauge symmetries, are expected to be respected by gravitational interactions ${ }^{1}$ and, therefore, they can prevent the presence of unwanted nonrenormalizable operators of lower dimensions. Thus, a $\mathbb{Z}_{N}$ preserving operator of some high dimension $D$ necessarily breaks $\mathrm{U}(1)_{X}$,

$$
\mathscr{L} \supset \frac{g}{M_{\mathrm{Pl}}^{D-4}} \sigma^{D}+\text { H.c. }, \quad(D>4)
$$

where $g=|g| e^{i \delta}$, with $|g|$ of order one; we assume the operator in eq. (2.16) is the one with lowest dimension with such a property. In that case, at leading order, a potential for the ALP is generated

$$
V(a) \approx-\frac{|g|}{2} \frac{v_{\sigma}^{D}}{\left(\sqrt{2} M_{\mathrm{Pl}}\right)^{D-4}} \cos \left[D \frac{a}{v_{\sigma}}+\delta\right] .
$$

This furnishes a mass to the ALP which can be very light for a sufficiently high $D$,

$$
m_{a} \approx|g|^{\frac{1}{2}} D \frac{v_{\sigma}}{\sqrt{2}} \times\left[\frac{v_{\sigma}}{\sqrt{2} M_{\mathrm{Pl}}}\right]^{\frac{D}{2}-2} .
$$

\footnotetext{
${ }^{1}$ The argument in ref. [9] is essentially that gravitational interactions must respect local symmetries and also any residual $\mathbb{Z}_{N}$ symmetry left in the effective theory after spontaneous breaking.
} 


\begin{tabular}{|c|c|c|}
\hline$D$ & $v_{\sigma}[\mathrm{GeV}]$ & $g_{a \gamma} / C_{a \gamma}\left[\mathrm{GeV}^{-1}\right]$ \\
\hline 7 & $6.04 \times 10^{8}$ & $1.92 \times 10^{-12}$ \\
8 & $2.44 \times 10^{10}$ & $4.76 \times 10^{-14}$ \\
9 & $3.43 \times 10^{11}$ & $3.39 \times 10^{-15}$ \\
10 & $2.50 \times 10^{12}$ & $4.64 \times 10^{-16}$ \\
11 & $1.18 \times 10^{13}$ & $9.87 \times 10^{-17}$ \\
12 & $4.07 \times 10^{13}$ & $2.86 \times 10^{-17}$ \\
13 & $1.12 \times 10^{14}$ & $1.03 \times 10^{-17}$ \\
\hline
\end{tabular}

Table 1. Necessary values for $v_{\sigma}$ to obtain $m_{a}=7.1 \mathrm{keV}$ in eq. (2.18) through the operator $\sigma^{D}$ in eq. (2.16), with $g=1$.

Intervals for the ALP parameters $\left(g_{a \gamma}, m_{a}\right)$ which can explain the anomalous Universe transparency for very energetic $\gamma$-ray [28-36], the soft X-ray excess from the Coma cluster [41, 42], and the X-ray line at $3.5 \mathrm{keV}[43,44,46]$, are shown in figure 2. It can be seen that there is a region where a set of parameters could explain both the anomalous Universe transparency and the soft X-ray excess from the Coma cluster. That region corresponds to $g_{a \gamma} \approx 10^{-11}-10^{-12} \mathrm{GeV}^{-1}$, which implies an ALP scale at the range

$$
\frac{v_{\sigma}}{C_{a \gamma}} \approx 10^{8}-10^{9} \mathrm{GeV},
$$

with $m_{a} \lesssim 10^{-12} \mathrm{eV}$. This requires that the $\mathrm{U}(1)_{X}$ breaking operators in eq. (2.16) should have dimensions of at least $D=11$ for $v_{\sigma}=10^{9} \mathrm{GeV}$, and $D=12$ for $v_{\sigma}=10^{10} \mathrm{GeV}$.

On the other hand, the region of parameters allowed for explaining the X-ray line at $3.5 \mathrm{keV}$ is disconnected from the previous region. Thus, if all these hinted phenomena are due to ALPs at least two different species of them are needed to exist. In order to explain the $3.5 \mathrm{keV}$ X-ray line through a decay of an ALP with mass $7.1 \mathrm{keV}$, the dimensionality of the operator in eq. (2.16) inducing such a mass depends on the scale $v_{\sigma}$. The values for the ALP scale $v_{\sigma}$ and the operator dimension $D$ inducing the correct mass with coupling in the range $g_{a \gamma} / C_{a \gamma} \approx 10^{-17}-10^{-12} \mathrm{GeV}^{-1}$ are shown in table 1 . For simplicity we take $g=1$. We see that the $\mathrm{U}(1)_{X}$ breaking operator needs to be of dimension 7 or larger.

Large portions of the ALP parameter space are expected to be probed directly by new experiments, and are already limited indirectly from astrophysical observations as shown in figure 2. Among the direct search experiments are the light-shining-through-wall experiment ALPS-II [56], the helioscope IAXO [57], and the observatories PIXIE [58] an PRISM [59]. Indirect astrophysical limits excluding portions of the parameter space are obtained from massive stars [74], the 1987A supernova [75-77], and quasar polarization [78, 79]. Since the present limit coming from the supernova 1987A [77] is stronger than the limits coming from quasar polarization, we do not show the latter in figure 2.

Next we present a general analysis of the symmetries and find requirements for an acceptable model. After that, we show specific constructions implementing the ISS mechanism with scales originating from the vev of one or more scalar fields, which contain ALPs having values of $\left(g_{a \gamma}, m_{a}\right)$ in the regions that could explain certain astrophysical phenomena. 


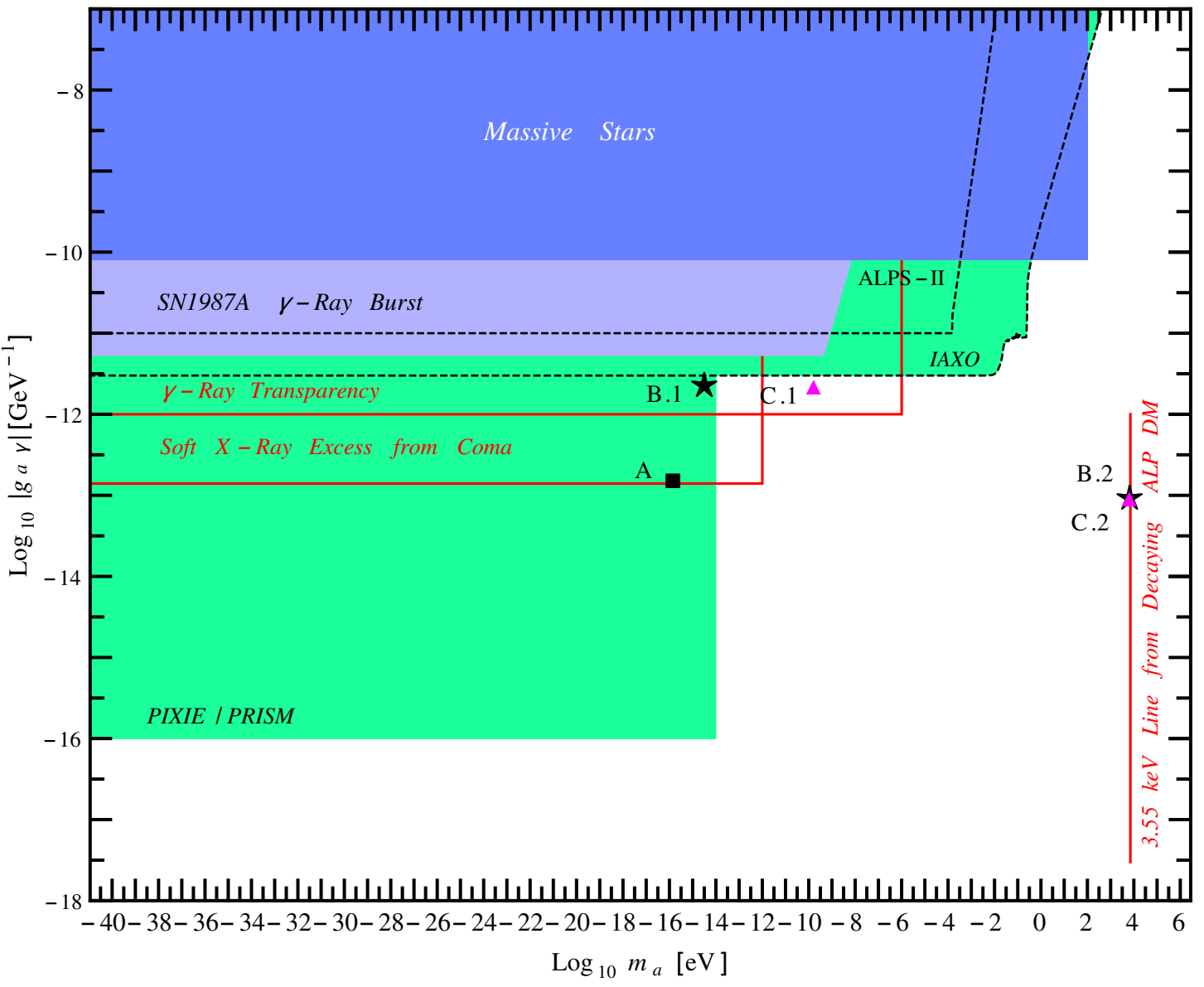

Figure 2. Figure adapted from ref. [26]. Values for the ALP coupling, $g_{a \gamma}$, and mass, $m_{a}$, required to explain indications of the anomalous Universe transparency for very energetic $\gamma$-ray [36], the soft X-ray excess from the Coma cluster [41, 42], and the X-ray line at $3.5 \mathrm{keV}[45,46,80,81]$, are inside the regions delimited by red lines. In green are the prospective regions to be reached by the light-shining-through-wall experiment ALPS-II [56], the helioscope IAXO [57], and the observatories PIXIE [58] an PRISM [59]. Also shown are astrophysical limits provided by: emission of ALP from massive stars representing an anomalous energy loss and shortening their helium-burning phase so that Cepheids could not be observed [74], non-observation of a $\gamma$-ray burst emitted by the core of the supernova SN 1987A in coincidence with their neutrinos burst arrival on Earth [75-77]. The benchmark points for the one ALP model of section 2.2 (A, square) and for the two-ALP models of section 2.3.1 (B.1 and B.2, stars) and section 2.3.2 (C.1 and C.2, triangles) are also shown.

\subsection{Symmetries of the model}

Two symmetries are essential in our construction: the continuous anomalous $\mathrm{U}(1)_{X}$ symmetry and the stabilizing discrete gauge symmetry $\mathbb{Z}_{N}$. Here we consider a single factor for simplicity but more factors can be equally considered. The continuous $\mathrm{U}(1)_{X}$ symmetry should arise accidentally from the conservation of $\mathbb{Z}_{N}$ at the intermediate ALP scale. The discrete symmetry, in turn, is assumed to be a remnant of a continuous gauge symmetry at higher energy scales, possibly at the GUT scale [9]. We will be concerned with the theory at intermediate scales and we will not attempt to trace the original continuous gauge 
symmetry as the possibilities are numerous. The anomalous nature of $\mathrm{U}(1)_{X}$ gives rise to the required ALP-photon coupling whereas the discrete symmetry $\mathbb{Z}_{N}$ should be anomaly free in the discrete sense [10-13] as required by its local nature.

Let us proceed to determine the generic aspects of the anomalous $\mathrm{U}(1)_{X}$ and the discrete $\mathbb{Z}_{N}$ symmetries. We will establish the following: $\mathrm{U}(1)_{X}$ acting on non-SM fields as in eq. (2.12) is an extension of the usual lepton number $\mathcal{L}$ and $\mathbb{Z}_{N}$ can be chosen as a discrete subgroup of some combination of $\mathcal{L}$ and the baryon number $\mathcal{B}$.

We start with $\mathrm{U}(1)_{X}$ and consider the Yukawa interactions of th SM:

$$
-\mathscr{L}_{\mathrm{Yuk}}^{\mathrm{SM}}=\bar{q}_{L} H d_{R}+\bar{q}_{L} \tilde{H} u_{R}+\bar{L} H l_{R},
$$

where we omit Yukawa couplings and family indices for simplicity. The SM fields are denoted as follows: $q_{i L}$ are the left-handed quark doublets; $u_{i R}$ and $d_{i R}$ are the righthanded quarks singlets; $l_{i R}$ are the right-handed lepton singlets; with $L_{i}$ and $H$ being respectively the left-handed lepton and Higgs doublets of eq. (2.7).

There are three family independent $\mathrm{U}(1)$ symmetries in eq. (2.20), coming from 3 independent constraints on 6 phases associated to 6 types of fields. They can be identified as hypercharge $Y$, baryon number $\mathcal{B}$ and lepton number $\mathcal{L}$. We adopt the usual assignment that the lepton fields $L_{i}, l_{i R}$ carry one unit of lepton number: $\mathcal{L}=1$.

We now consider the addition of the right-handed neutrino fields $N_{R}, S_{R}$, necessary for the ISS mechanism, and also the complex singlet scalar $\sigma$ whose vev sets the neutrino mass scales $M, \mu$. These three complex fields contribute to the Lagrangian in eq. (2.7), containing three terms, and no additional U(1) symmetry appear. These new fields do not carry neither hypercharge nor baryon number, the latter following from the absence of interactions with quarks. Thus they carry an extended lepton number. In particular, because of the first term in eq. (2.7), $N_{i R}$ carries the same lepton number as $L_{i}$. If we denote by $a, d$ the lepton number of $S_{R}$ and $\sigma$, respectively, the last two terms in eq. (2.7) result in

$$
d=\left(p-\frac{1}{2} q\right)^{-1}, \quad a=\frac{1}{2} q d,
$$

where $p \neq q / 2$ is required from independency of constraints. If we exchange $\sigma$ by $\sigma^{*}$ in the last term in eq. (2.7), it is sufficient to consider negative $q \rightarrow-q$ in all equations. We conventionally adopt positive $p$.

At last, the new vector-like fermion fields $E_{L}, E_{R}$ have the same electric charge as $l_{i R}$ and its hypercharge is defined. They interact through

$$
-\mathscr{L} \supset k_{i} \frac{\sigma^{r}}{M_{\mathrm{Pl}}^{r}} \bar{L}_{i} H E_{R}+k_{E} \frac{\sigma^{s}}{M_{\mathrm{Pl}}^{s-1}} \bar{E}_{L} E_{R}
$$

with small integers $r, s$; note that $s$ cannot be zero to generate an anomalous symmetry but it should also obey $|s| \leq 3$ to induce sufficiently large masses for $E$, for ALP scales in the range in eq. (2.5). Roughly speaking, the value of $r$ determine the life time of the charged lepton $E$ : the larger the value of $r$, the longer the life time of the $E$ particle. If $r$ is too large, the first term in eq. (2.22) becomes negligible, and thus the $E$ exotic lepton will be a stable charged particle which is cosmologically problematic, unless its mass is $\lesssim \mathrm{TeV}$ [82]. Another constraint comes from searches for long-lived charged particles in pp collisions [83]. We will discuss this in more detail below. 


\begin{tabular}{|c|c|c|c|c|c|c|c|c|c|c|c|}
\hline & $q_{i L}$ & $d_{i R}$ & $u_{i R}$ & $H$ & $L_{i}$ & $l_{i R}$ & $N_{i R}$ & $S_{i R}$ & $E_{L}$ & $E_{R}$ & $\sigma$ \\
\hline$Y$ & $\frac{1}{6}$ & $-\frac{1}{3}$ & $\frac{2}{3}$ & $\frac{1}{2}$ & $-\frac{1}{2}$ & -1 & 0 & 0 & -1 & -1 & 0 \\
\hline $\mathcal{B}$ & $\frac{1}{3}$ & $\frac{1}{3}$ & $\frac{1}{3}$ & 0 & 0 & 0 & 0 & 0 & 0 & 0 & 0 \\
\hline $\mathcal{L}$ & 0 & 0 & 0 & 0 & 1 & 1 & 1 & $a$ & $b$ & $c$ & $d$ \\
\hline
\end{tabular}

Table 2. Continuous symmetries of the model. Charges $a, b, c, d$ are determined by eqs. (2.21) and (2.23).

The two interaction terms in eq. (2.22) then determine the lepton numbers of $E_{L}, E_{R}$ without affecting the number of symmetries. If we denote the lepton number of $E_{L}, E_{R}$ by $b, c$ respectively, we obtain explicitly

$$
b-c=s d, \quad c=1-r d .
$$

A negative $s, r$ in eq. (2.23) may account for the simple exchange $\sigma \rightarrow \sigma^{*}$ in the respective terms. The final set of $\mathrm{U}(1)$ symmetries of the model consists of $Y, \mathcal{B}, \mathcal{L}$ generated by charges listed in table 2 . The $\mathcal{L}$-charges $a, b, c, d$ of fields $S_{R}, E_{L}, E_{R}, \sigma$ were determined by eqs. (2.21) and (2.23). Since $\mathcal{B}$, and obviously $Y$, are not anomalous with respect to electromagnetism, the anomalous symmetry $\mathrm{U}(1)_{X}$ can be chosen to be generated by some multiple of the extended lepton number $\mathcal{L}$. Specifically, since the anomaly is proportional to $b-c, s$ cannot be zero in view of eq. (2.23).

Concerning the discrete symmetry $\mathbb{Z}_{N}$, the following anomaly cancellation conditions should hold from the effective point of view [10-16]:

$$
A_{2}=A_{3}=A_{\text {grav }}=0 \bmod N / 2,
$$

where $A_{2}, A_{3}, A_{\text {grav }}$ are the anomaly coefficients associated with $\left[\mathrm{SU}(2)_{L}\right]^{2} \times \mathbb{Z}_{N},\left[\mathrm{SU}(3)_{c}\right]^{2} \times$ $\mathbb{Z}_{N}$ and [gravity $]^{2} \times \mathbb{Z}_{N}$, respectively. We ignore the anomaly associated to $\left[\mathrm{U}(1)_{Y}\right]^{2} \times \mathbb{Z}_{N}$ because it does not furnish useful low energy constraints [11].

We write the action of $\mathbb{Z}_{N}$ as

$$
\psi_{k} \rightarrow e^{i 2 \pi Z_{k} / N} \psi_{k}
$$

with discrete charges $Z_{k}=Z\left(\psi_{k}\right)=0,1, \cdots, N-1$. Given that $\mathrm{U}(1)_{Y}$ is anomaly free by construction, and its imposition has no effect on undesirable operators, we can consider our discrete $\mathbb{Z}_{N}$ to be a discrete subgroup of the rest of the symmetries in table $2[22,24]$ :

$$
Z=c_{1} \mathcal{B}+c_{2} \mathcal{L}
$$

where $c_{i}$ should be rational numbers that makes all $Z$ charges integers. To avoid redundancy, we can adopt $c_{1}=n_{1} 3$ and $c_{2}=n_{2} \tilde{c}_{2}$, where $n_{1}, n_{2}=1, \ldots, N-1$ and $\tilde{c}_{2}$ is the smallest integer that makes all $\mathcal{L}$-charges integer and coprime. The factor 3 in $c_{1}$ appears because only $3 \mathcal{B}$ is made of integers. If $N$ is not a prime, we also need to discard values for $n_{i}$ that makes $c_{1} \mathcal{B}$ or $c_{2} \mathcal{L}$ to have a common factor that divides $N$. The latter case implies only a subgroup of $\mathbb{Z}_{N}$ is realized. 
We can now calculate the anomaly coefficients as

$$
A_{i}(Z)=c_{1} A_{i}(\mathcal{B})+c_{2} A_{i}(\mathcal{L})
$$

where

$$
\begin{aligned}
A_{i}(\mathcal{B}) & =\left(\frac{3}{2}, 0,0\right) \\
A_{i}(\mathcal{L}) & =\left(\frac{3}{2}, 0,-3 a+b-c\right),
\end{aligned}
$$

with $i=2,3$, grav, respectively. In special, the gravitational anomaly only depends on $\mathcal{L}$ and we can write

$$
A_{\text {grav }}(Z)=c_{2}\left(s-\frac{3}{2} q\right) d,
$$

where eqs. (2.21) and (2.23) are used. We can see the well-known result that $\mathcal{B}-\mathcal{L}$ is anomaly free for $a=0$ and $b=c$, which corresponds to the SM with three right-handed neutrinos; see e.g. ref. [84]. Therefore, any discrete subgroup of $\mathcal{B}-\mathcal{L}$ will have $A_{2}$ and $A_{3}$ automatically canceled. However, due to its discrete nature, eq. (2.24), distinct combinations of $\mathcal{B}$ and $\mathcal{L}$ can be also anomaly free as well. One can also check, there is no intrinsic discrete symmetry besides subgroups of combinations of $Y, \mathcal{B}, \mathcal{L}$; use, e.g., the Smith Normal Form method [85, 86].

To summarize, we seek SM extensions defined by eqs. (2.7) and (2.22), with ALP decay constant $v_{\sigma}$, integers $(p, q, r, s)$ and discrete symmetry $\mathbb{Z}_{N}$ obeying the following restrictions:

1. one ALP is present that couples to photons and explains one or more astrophysical phenomena indicated in figure 2 ;

2. the correct mass scales for the ISS mechanism are generated by $v_{\sigma}$;

3. the ISS mechanism is stabilized by $\mathbb{Z}_{N}$;

4. the mass of the heavy lepton $E$ is larger than the electroweak scale: $M_{E} \gtrsim v_{\mathrm{w}}$.

5. There is no discrete anomaly for $\mathbb{Z}_{N}$.

Extensions to more than one ALP should obey analogous conditions.

The conditions for items 1, 2, 4 and 5 have already been discussed. To summarize conditions 1 and 4 , it is necessary to have $0<|s| \leq 3$ and the singlet $\sigma$ should be charged by $\mathrm{U}(1)_{X}$; and $|s|=3$ is possible only if the ALP scale is high, $v_{\sigma} \gtrsim 10^{13} \mathrm{GeV}$. The stability of the ISS mechanism, condition 3, requires the following: $N_{R}, S_{R}$ should be charged under $\mathbb{Z}_{N}$ to avoid the direct Majorana terms $\bar{N}_{R} N_{R}^{c}$ and $\bar{S}_{R} S_{R}^{c}$. Moreover, $\mathbb{Z}_{N}$ charges should prevent the appearance of operators $\sigma^{n} \bar{S}_{R} S_{R}^{c}, \sigma^{n} \bar{S}_{R} N_{R}^{c}, \sigma^{n} \bar{N}_{R} N_{R}^{c}, \sigma^{n} \bar{L} \tilde{H} S_{R}$ with dimension lower than the ones inducing the correct ISS scales in condition 2; and the same applies for operators that replaces $\sigma$ with $\sigma^{*}$. Specifically, any operator of the form $\sigma^{n} \bar{N}_{R} N_{R}^{c}$ or $\sigma^{n^{\prime}} \bar{L} \tilde{H} S_{R}$ disrupts the zeros in the ISS texture in eq. (2.2). Nevertheless, the mass matrix in eq. (2.3) is the leading contribution as long as $\left|n^{\prime}\right|>|q|-p,|n|>2 p-|q|$ 
and $|n|+\left|n^{\prime}\right|>|q|$; order of magnitude conditions can be extracted from subleading contributions in the seesaw formula, cf. $[62,63,87]$. To guarantee that these contribution are negligible, we require a more strict condition: $|n| \geq 4$ and $\left|n^{\prime}\right| \geq 3$. The presence of these dangerous operators can be traced from their $\mathcal{L}$-charges:

$$
\bar{L} \tilde{H} S_{R} \sim(q-p) d, \quad \bar{N}_{R}^{c} N_{R} \sim(2 p-q) d,
$$

where we have conveniently written the charges in terms of the charge of $\sigma \sim d$. Therefore, the combinations $q-p$ and $2 p-q$ control the coupling of these operators to powers of $\sigma$ and some combinations of $p, q$ can be readily excluded in the case of one singlet. For example $(p, q)=(2,3)$, is excluded because it allows both operators in eq. (2.30) to couple to $\sigma^{*}$. Generically, it is more interesting to have negative $q$ when $p \neq 0$.

\subsection{Model with one ALP}

We focus first on a model which according to our previous considerations could explain the Universe transparency and the soft X-ray excess from the Coma cluster. As we pointed out in figure 2, there is an overlap in the parameter space and for certain values of $\left(g_{a \gamma}, m_{a}\right)$ the same ALP could be responsible for both phenomena. In addition, to obtain correct order of magnitude parameters for the ISS, we take the singlet vev to be within the first interval in eq. (2.10), corresponding to the case $(p, q)=(2,3)$. This choice leads to an ALP-photon coupling constant in eq. (2.14) in the range

$$
\frac{g_{a \gamma}}{C_{a \gamma}} \approx 2.1 \times 10^{-14}-5.3 \times 10^{-14} \mathrm{GeV}^{-1} .
$$

For a coefficient $C_{a \gamma}$ of order one, the value of $g_{a \gamma}$ would be outside the region required to explain the Universe transparency. But it would be still possible to explain the soft X-ray excess from the Coma cluster if $C_{a \gamma} \sim 5$ and the ALP mass is restricted to $m_{a} \leq$ $10^{-12} \mathrm{eV}[41,42]$.

We choose the Lagrangian given by eqs. (2.7) and (2.22), with $(p, q)=(2,-3),(r, s)=$ $(1,2)$. For convenience, we write explicitly only the terms that depend on the singlet $\sigma$ :

$$
\begin{aligned}
-\mathcal{L} \supset & \eta_{i j} \frac{\sigma^{2}}{M_{\mathrm{Pl}}} \bar{S}_{i R}\left(N_{j R}\right)^{c}+\frac{1}{2} \zeta_{i j} \frac{\sigma^{* 3}}{M_{\mathrm{Pl}}^{2}} \bar{S}_{i R}\left(S_{j R}\right)^{c} \\
+ & k_{i} \frac{\sigma}{M_{\mathrm{Pl}}} \bar{L}_{i} H E_{R}+k_{E} \frac{\sigma^{2}}{M_{\mathrm{Pl}}} \bar{E}_{L} E_{R}+\text { H.c. }
\end{aligned}
$$

The choices above lead to $d=2 / 7$ in eq. (2.21) for the lepton number of $\sigma$. As a consequence, only $7 \mathcal{L}$ has all charges integer and the stability of the ISS mechanism requires either a discrete symmetry $\mathbb{Z}_{11}$ or $\mathbb{Z}_{13}$. Other choices allow operators of the forms $\bar{L} \tilde{H} S_{R} \sigma^{n}, \bar{N}_{R}^{c} N_{R} \sigma^{n}$ with dimensions that are too low. However, because of gravitational anomaly, we choose $\mathbb{Z}_{13}$ as it can be seen below.

The simplest possibility for the anomaly free discrete $\mathbb{Z}_{13}$ symmetry is

$$
Z=6 \mathcal{B}+7 \mathcal{L}
$$




\begin{tabular}{|c|c|c|c|c|c|c|c|c|c|c|c|}
\hline $\mathbb{Z}_{13}$ & $q_{i L}$ & $d_{i R}$ & $u_{i R}$ & $H$ & $L_{i}$ & $l_{i R}$ & $N_{i R}$ & $S_{i R}$ & $E_{L}$ & $E_{R}$ & $\sigma$ \\
\hline$Z$ & 2 & 2 & 2 & 0 & 7 & 7 & 7 & -3 & 9 & 5 & 2 \\
\hline
\end{tabular}

Table 3. $\mathbb{Z}_{13}$ charges $Z_{i}=(6 \mathcal{B}+7 \mathcal{L})_{i}$ in the notation of eq. (2.25).

\begin{tabular}{|c|c|c|c|c|c|c|c|c|c|c|c|}
\hline $\mathrm{U}(1)_{X}$ & $q_{i L}$ & $d_{i R}$ & $u_{i R}$ & $H$ & $L_{i}$ & $l_{i R}$ & $N_{i R}$ & $S_{i R}$ & $E_{L}$ & $E_{R}$ & $\sigma$ \\
\hline$X$ & 0 & 0 & 0 & 0 & $\frac{7}{2}$ & $\frac{7}{2}$ & $\frac{7}{2}$ & $-\frac{3}{2}$ & $\frac{9}{2}$ & $\frac{5}{2}$ & 1 \\
\hline
\end{tabular}

Table 4. $\mathrm{U}(1)_{X}$ charge assignments for the fields in eq. (2.32).

where the coefficients in eq. (2.26) are chosen as follows: $c_{2}=7$ is kept and $c_{1}=6$ is chosen from the cancellation of mixed $\left[\mathrm{SU}(2)_{L}\right]^{2} \times \mathbb{Z}_{13}$ anomaly. The explicit anomaly coefficients for $\mathbb{Z}_{13}$ are

$$
A_{2}=39 / 2, \quad A_{3}=0, \quad A_{\text {grav }}=13,
$$

which are all zero modulo $N / 2=13 / 2$. Hence, since the gravitational anomaly only depends on $\mathcal{L}$, we can see only $\mathbb{Z}_{13}$ is anomaly free and we can discard $\mathbb{Z}_{11}$. The ISS mechanism is also stable as the lowest order $\mathbb{Z}_{13}$ invariant operators that could disrupt the mechanism are $\sigma^{6} \bar{N}_{R}^{c} N_{R}, \sigma^{5} \bar{L} \tilde{H} S_{R}$. The explicit charges $Z_{i}$ for each field can be seen in table 3. Note that, for the SM model fields, the $\mathbb{Z}_{13}$ charges are equivalent to $6(\mathcal{B}-\mathcal{L})$ modulo 13.

We can see that the imposition of the $\mathbb{Z}_{13}$ symmetry in eq. (2.32) successfully leads to an accidental $\mathrm{U}(1)_{X}$ symmetry, corresponding to the extended lepton number $\mathcal{L}$ in table 2 , with charges conveniently rescaled in table 4 to give $X_{\sigma}=1$. Such a symmetry coincides with the usual lepton number for the SM fields, but it is anomalous for $E_{L}, E_{R}$ fields. The accidental $\mathrm{U}(1)_{X}$ symmetry is only approximately conserved because it is explicitly broken by higher dimensional $\mathbb{Z}_{13}$ invariant operators suppressed by the Planck scale.

As the singlet field $\sigma$ acquires a large vev, $v_{\sigma} \sim 3 \times 10^{10} \mathrm{GeV}$, the anomalous $\mathrm{U}(1)_{X}$ is spontaneously broken, making its phase field $a(x)$ in eq. (2.6) a pseudo Nambu-Goldstone boson. The singlet vev will also generate the ISS mass parameters in eq. (2.8) in the correct order of magnitude. The characteristic shift symmetry for $a(x)$ is broken by $\mathbb{Z}_{13}$ invariant operators of the form in eq. (2.16), where $\sigma^{13}$ is the operator of smallest dimension. The latter operator gives the dominant contribution in eq. (2.18) for the ALP mass, with magnitude

$$
m_{a} \simeq 1.58 \times 10^{-16} \mathrm{eV}|g|^{\frac{1}{2}}\left(\frac{v_{\sigma}}{3 \times 10^{10} \mathrm{GeV}}\right)^{5.5} .
$$

With the charges in table 4 and from eq. (2.15), we can readily calculate the anomaly coefficient $C_{a \gamma}=4$ and the ALP-photon coupling,

$$
g_{a \gamma} \simeq 1.5 \times 10^{-13}\left(\frac{3 \times 10^{10} \mathrm{GeV}}{v_{\sigma}}\right) \mathrm{GeV}^{-1} .
$$

The benchmark point for this model, named $\mathbf{A}$, is shown in figure 2. Although the ALP in this model may explain the soft X-ray excess from the Coma cluster, it is out of the projected regions for searches of the ALP-II [56] and IAXO [57] experiments. But it is inside the region that will be probed by the planned observatories PIXIE/PRISM $[58,59]$. 
Since all $\mathrm{U}(1)_{X}$ and $\mathbb{Z}_{13}$ charges are family blind, our model does not lead to specific predictions for the neutrino flavor structure (family dependent $\mathrm{U}(1)$ symmetries leading to axions has been considered in, e.g., refs. [88, 89]). Only the order of magnitude for the absolute mass scale is obtained through eq. (2.11) as

$$
m_{\nu} \approx\left[y^{\top} \eta^{-1} \zeta\left(\eta^{\top}\right)^{-1} y\right] \times 1.4 \times 10^{3} \mathrm{eV}
$$

for $v_{\mathrm{w}}=246 \mathrm{GeV}$ and $v_{\sigma}=3 \times 10^{10} \mathrm{GeV}$. The mass matrices in eq. (2.8) for the ISS mechanism acquire quite natural values as

$$
M=\eta \times 187 \mathrm{GeV}, \quad \mu=\zeta \times 1.6 \mathrm{keV} .
$$

Typically we will need the matrix entries of $\eta$ to be larger than one, e.g., $\eta \sim 5$, and $y \ll 1$, to evade lepton flavor violation [64-66] and direct detection constraints [67,68] as well as to maintain the validity of the seesaw formula in eq. (2.4). On the other hand, $\zeta$ can be of order one or smaller. To obtain light neutrino masses in the sub-eV range, we need small Yukawa coupling, $y \lesssim 0.1$ or smaller. The largest eigenvalue of the combination of matrices inside brackets in eq. (2.37) needs to be at most around $10^{-4}$.

From table 4, we note that the charged lepton $E$ is the only one that contributes to the coefficient $C_{a \gamma}$ because $E$ has electric charge different from zero and $X_{E_{L}} \neq X_{E_{R}}$. In this model, its mass comes from the term $k_{E} \frac{\sigma^{2}}{M_{\mathrm{Pl}}} \bar{E}_{L} E_{R}$ in eq. (2.32). When $\sigma$ gains a vev, $E$ obtains a mass, $M_{E}=k_{E} \frac{v_{\sigma}^{2}}{2 M_{\mathrm{Pl}}} \approx k_{E} \times 187 \mathrm{GeV}$. Therefore, we typically need $k_{E}$ to be larger than one to avoid the current lower limit of $574 \mathrm{GeV}$ on the mass of new charged leptons [83]. To be more specific, the latter limit applies for charged long-lived heavy lepton with lifetime greater than a few nanoseconds, because these particles can travel distances comparable to the size of modern detectors and thus appear to be stable. However, in this model $E$ can decay into $e_{i}$ and $h^{0}$, with $i=e, \mu, \tau$ and $h^{0}$ being the Higgs boson with mass of $m_{h^{0}}=125 \mathrm{GeV}$. This decay is induced by the term $k_{i} \frac{\sigma}{M_{\mathrm{Pl}}} \bar{L}_{i} H E_{R}$ in eq. (2.32). Estimating the lifetime of $E, \tau_{E}$, we find that for $m_{E}>m_{h^{0}}, \tau_{E}$ can be written as

$$
\tau_{E} \simeq \frac{16 \pi}{3 \lambda^{2}} \frac{m_{E}^{3}}{\left(m_{E}^{2}-m_{h^{0}}^{2}\right)^{2}} \times 6.5822 \times 10^{-25} \mathrm{~s},
$$

where we have neglected the masses of the SM leptons, i.e., $m_{e}, m_{\mu}, m_{\tau} \rightarrow 0$. We have also considered that $k_{e}=k_{\mu}=k_{\tau}$. The factor $\lambda$ in eq. (2.39) is $\frac{k_{i} v_{\sigma}}{\sqrt{2} M_{\mathrm{Pl}}}$. Taking $k_{e}=k_{\mu}=k_{\tau}=1$, we find that for $m_{E} \gtrsim 250 \mathrm{GeV}$, the charged lepton $E$ has a lifetime smaller than $10^{-9} \mathrm{~s}$. Therefore, the lower limit of $574 \mathrm{GeV}$ does not apply and order one values for $k_{E}$ are still allowed.

Concerning other possibilities, a few remarks are in order. Considering the SM augmented by only one singlet scalar $\sigma$ and fermion fields $N_{R}, S_{R}, E_{L}, E_{R}$ through eqs. (2.7) and (2.22),

- there is no other model that contains one ALP capable of explaining the transparency of the Universe or the soft X-ray excess in the Coma cluster;

- it is also not possible to find models featuring an ALP with mass $m_{a} \sim 7.1 \mathrm{keV}$, which can explain the $3.55 \mathrm{keV}$ X-ray line through the decay of the ALP into two photons $[45,46]$. 
Other choices for the powers $(p, q)$, such as $(p, q)=(3, \pm 5)$, and for the discrete symmetry $\mathbb{Z}_{N}$ do not comply with one or more of the restrictions explained in the end of section 2.1: (i) generation of correct mass scales for the ISS mechanism and (ii) stabilization of the ISS mechanism and (iii) cancellation of discrete anomalies. Many possibilities are excluded by (ii) because they allow low-dimensional operators $\sigma^{n}$ to couple to $\bar{N}_{R}^{c} N_{R}$ or $\bar{L} \tilde{H} S_{R}$. Further restriction comes from the gravitational anomaly cancellation, (iii), and only the $\mathbb{Z}_{13}$ symmetry model survives.

\subsection{Models with two ALPs}

Here we extend the previous setting and seek models featuring two ALPs that can explain the excess of X-ray photons in the $3.5 \mathrm{keV}$ line, in addition to the transparency of the Universe for ultra energetic gamma rays and the soft X-ray excess from the Coma cluster. As we have previously discussed and shown in figure 2, at least two ALPs are necessary to explain these three phenomena. Hence, in addition to the singlet $\sigma$, we introduce another SM singlet $\sigma^{\prime}$ which will host a second ALP $a^{\prime}$. Now, two energy scales, $v_{\sigma}=\sqrt{2}\langle\sigma\rangle$ and $v_{\sigma^{\prime}}=\sqrt{2}\left\langle\sigma^{\prime}\right\rangle$, will govern the physics of these ALPs. Since the ALP $a^{\prime}$ should also couple to photons, the singlet $\sigma^{\prime}$ should be charged under another anomalous symmetry $\mathrm{U}(1)_{X^{\prime}}$, which follows accidentally from a second $\mathbb{Z}_{N^{\prime}}$ symmetry.

Let us choose $a$ to be the ALP of $7.1 \mathrm{keV}$ mass that explains the $3.55 \mathrm{keV}$ X-ray line. The possible values for $v_{\sigma}$ and the $\mathbb{Z}_{N}$ symmetry that are needed can be seen in table 1 for $|g|=1$. The possible values for $v_{\sigma^{\prime}}$ are then restricted by eq. $(2.19), v_{\sigma^{\prime}} \approx 10^{9} \mathrm{GeV}$, and $\sigma^{\prime}$ should be protected by a discrete symmetry $\mathbb{Z}_{N^{\prime}}$ with $N^{\prime} \geq 11$. Note that the two ALP scales do not mix in our models, following the ones proposed in ref. [26]. Models where only $\sigma$ (or $\sigma^{\prime}$ ) couples to $N_{R}, S_{R}$ are excluded from the considerations of the previous section. We need that both $v_{\sigma}$ and $v_{\sigma^{\prime}}$ generate the ISS mass scales. However, we were unable to find a plausible model that could satisfy all conditions listed in the end of section 2.1. Thus we present in the following, two models that satisfy almost all criteria.

The general Lagrangian we will consider is composed of the usual Yukawa interactions in eqs. (2.20) and (2.7), with terms depending on the scalar singlets modified to

$$
\begin{aligned}
-\mathscr{L} \supset & \frac{\sigma^{p} \sigma^{\prime p^{\prime}}}{M_{\mathrm{Pl}}^{p+p^{\prime}-1}} \overline{S_{R}} \eta N_{R}^{c}+\frac{\sigma^{q} \sigma^{\prime q^{\prime}}}{M_{\mathrm{Pl}}^{q+q^{\prime}-1}} \overline{S_{R}} \zeta S_{R}^{c} \\
& +k_{i} \frac{\sigma^{r} \sigma^{\prime \prime^{\prime}}}{M_{\mathrm{Pl}}^{r+r^{\prime}}} \overline{L_{i}} H E_{R}+k_{E} \frac{\sigma^{s}{\sigma^{\prime s^{\prime}}}_{M_{\mathrm{Pl}}^{s+s^{\prime}-1}} \overline{E_{L}} E_{R}+\text { H.c. }}{}
\end{aligned}
$$

Many restrictions on the integers $(p, q, r, s)$ discussed in section 2.1 and in the beginning of section 2 are now valid for the sum of unprimed and primed variables. For example, the restriction in eq. (2.9) should be now adapted to $p+p^{\prime}=2,3$ and $|q|+\left|q^{\prime}\right|=3,4,5$, where we conventionally take $p, p^{\prime}$ to be positive. Likewise, condition 4 in the end of section 2.1, for low ALP scales, is now $|s|+\left|s^{\prime}\right| \leq 2$, which leads to $s= \pm 1$ and $s^{\prime}= \pm 1$. We also see that the number of symmetries are consistent: there is one more field $\sigma^{\prime}$ for the same number of constraints but we need one more anomalous symmetry. Given that the fields beyond the SM only couple to leptons, we can still consider $X$ and $X^{\prime}$ proportional to two 
extended lepton numbers $\mathcal{L}$ and $\mathcal{L}^{\prime}$. Additionally, all formulas for $(p, q, r, s)$ in section 2 still apply considering that $\sigma$ is only charged under $\mathcal{L}$ while $\sigma^{\prime}$ is only charged under $\mathcal{L}^{\prime}$. Thus the same formulas applies for the primed $\left(p^{\prime}, q^{\prime}, r^{\prime}, s^{\prime}\right)$ as well, depending now on $\mathcal{L}^{\prime}$-charges $\left(a^{\prime}, b^{\prime}, c^{\prime}, d^{\prime}\right)$ of $S_{R}, E_{L}, E_{R}, \sigma^{\prime}$, respectively.

\subsubsection{Model I}

The first model gives up the cancellation of the gravitational anomaly for one of the discrete symmetries $\mathbb{Z}_{N}$ or $\mathbb{Z}_{N^{\prime}}$. The anomaly can be easily canceled by the addition of one or more fermions that are singlets of the SM but do not contribute to the phenomena discussed in this paper. The model also gives rise to a small scale for $\mu$.

We consider the interaction terms for the singlet fields to be

$$
\begin{aligned}
-\mathscr{L} \supset & \eta_{i j} \frac{\sigma^{2}}{M_{\mathrm{Pl}}} \overline{S_{R i}} N_{R j}^{c}+\zeta_{i j} \frac{\sigma^{* * 3}}{M_{\mathrm{Pl}}^{2}} \overline{S_{R i}} S_{R j}^{c} \\
& +k_{i} \frac{\sigma \sigma^{\prime}}{M_{\mathrm{Pl}}^{2}} \overline{L_{i}} H E_{R}+k_{E} \frac{\sigma \sigma^{\prime}}{M_{\mathrm{Pl}}} \overline{E_{L}} E_{R}+\text { H.c. }
\end{aligned}
$$

The Lagrangian has the form in eq. (2.40) with $(p, q)=(2,0)$ and $\left(p^{\prime}, q^{\prime}\right)=(0,-3)$, whereas $(r, s)=\left(r^{\prime}, s^{\prime}\right)=(1,1)$. We choose $v_{\sigma} \approx 2.44 \times 10^{10} \mathrm{GeV}$ to accommodate the correct scale for $M$ and generate the ALP mass of $m_{a}=7.1 \mathrm{keV}$, which should be protected by a $\mathbb{Z}_{8}$ symmetry. We protect $\sigma^{\prime}$ with a symmetry $\mathbb{Z}_{11}$ so that the whole model has a symmetry $\mathbb{Z}_{8} \times \mathbb{Z}_{11}$.

Let us proceed to find the symmetry $\mathbb{Z}_{8} \times \mathbb{Z}_{11}$. Eq. (2.21) determines $(d, a)=(1 / 2,0)$ and $\left(d^{\prime}, a^{\prime}\right)=(2 / 3,-1)$. Therefore $c_{2}=2$ and $c_{2}^{\prime}=3$ makes all charges of $c_{2} \mathcal{L}$ and $c_{2}^{\prime} \mathcal{L}^{\prime}$ integers and we can calculate the gravitational anomaly from eq. (2.29):

$$
A_{\text {grav }}\left(c_{2} \mathcal{L}\right)=1, \quad A_{\text {grav }}\left(c_{2}^{\prime} \mathcal{L}^{\prime}\right)=11
$$

It is clear that the gravitational anomaly for $c_{2} \mathcal{L}$ does not cancel for any $\mathbb{Z}_{N}$, except $\mathbb{Z}_{2}$. Note that we can not use $c_{2}=2 \times 4$ to cancel the gravitational anomaly because $8 \mathcal{L}$ only generates $\mathbb{Z}_{2}$. Therefore, we assume such a gravitational anomaly is canceled by additional fermion fields and we adopt $\mathbb{Z}_{8}$ generated by $Z=6 \mathcal{B}+2 \mathcal{L}$ (we could have adopted $Z=6 \mathcal{B}-6 \mathcal{L}$ as well). This choice cancels the anomaly of $A_{2}(Z)$. Analogously, we choose the $\mathbb{Z}_{11}$ generator as $Z^{\prime}=-3 \mathcal{B}+3 \mathcal{L}^{\prime}$. We show the explicit charges in table 5 . The charges for the anomalous $\mathrm{U}(1)_{X}$ and $\mathrm{U}(1)_{X^{\prime}}$ are presented in table 6 .

This model yields $C_{a \gamma}=C_{a^{\prime} \gamma}=2$, cf. eq. (2.15), which leads to the desired ALP-photon couplings

$$
\begin{gathered}
g_{a \gamma}=\frac{\alpha}{2 \pi v_{\sigma}} C_{a \gamma} \approx 9.52 \times 10^{-14} \mathrm{GeV}^{-1}, \\
g_{a^{\prime} \gamma}=\frac{\alpha}{2 \pi v_{\sigma^{\prime}}} C_{a^{\prime} \gamma} \approx 2.32 \times 10^{-12} \mathrm{GeV}^{-1},
\end{gathered}
$$

for $v_{\sigma} \approx 2.44 \times 10^{10} \mathrm{GeV}$ and $v_{\sigma^{\prime}} \approx 10^{9} \mathrm{GeV}$. The ALP masses are given by

$$
m_{a} \approx|g| \times 7.1 \mathrm{keV}, \quad m_{a^{\prime}} \approx\left|g^{\prime}\right| \times 3.41 \times 10^{-15} \mathrm{eV} .
$$

Benchmark points for ALPs $a$ and $a^{\prime}$ are marked as B.1 and B.2 in figure 2 for $|g|=\left|g^{\prime}\right|=1$. 


\begin{tabular}{|c|c|c|c|c|c|c|c|c|c|c|c|c|}
\hline & $q_{i L}$ & $d_{i R}$ & $u_{i R}$ & $H$ & $L_{i}$ & $l_{i R}$ & $N_{i R}$ & $S_{i R}$ & $E_{L}$ & $E_{R}$ & $\sigma$ & $\sigma^{\prime}$ \\
\hline $\mathbb{Z}_{8}$ & 2 & 2 & 2 & 0 & 2 & 2 & 2 & 0 & 2 & 1 & 1 & 0 \\
\hline $\mathbb{Z}_{11}$ & -1 & -1 & -1 & 0 & 3 & 3 & 3 & -3 & 3 & 1 & 0 & 2 \\
\hline
\end{tabular}

Table 5. $\mathbb{Z}_{8} \times \mathbb{Z}_{11}$ charges in the notation in eq. (2.25).

\begin{tabular}{|c|c|c|c|c|c|c|c|c|c|c|c|c|}
\hline & $q_{i L}$ & $d_{i R}$ & $u_{i R}$ & $H$ & $L_{i}$ & $l_{i R}$ & $N_{i R}$ & $S_{i R}$ & $E_{L}$ & $E_{R}$ & $\sigma$ & $\sigma^{\prime}$ \\
\hline$X$ & 0 & 0 & 0 & 0 & 2 & 2 & 2 & 0 & 2 & 1 & 1 & 0 \\
\hline$X^{\prime}$ & 0 & 0 & 0 & 0 & $\frac{3}{2}$ & $\frac{3}{2}$ & $\frac{3}{2}$ & $-\frac{3}{2}$ & $\frac{3}{2}$ & $\frac{1}{2}$ & 0 & 1 \\
\hline
\end{tabular}

Table 6. Charges for $\mathrm{U}(1)_{X}$ and $\mathrm{U}(1)_{X^{\prime}}$.

The induced neutrino mass matrices have magnitude

$$
M=\eta \times 124 \mathrm{GeV}, \quad \mu=\zeta \times 0.061 \mathrm{eV},
$$

which leads to the light neutrino mass matrix

$$
m_{\nu}=\left[y^{\top} \eta^{-1} \zeta\left(\eta^{\top}\right)^{-1} y\right] \times 0.12 \mathrm{eV} .
$$

Analogously to the model of section 2.2, we typically need $\eta$ to have entries with magnitude larger than one while the Yukawa coefficients need to be smaller than one, so that $\epsilon=$ $m_{D} M^{-1}$ has small entries. The matrix $\zeta$ can have entries of order one or smaller but we can see the scale generated by $\sigma^{\prime}$ is smaller than the one generated in eq. (2.38). The ISS mechanism is stable as the new operators of lowest order are $N_{R}^{2}\left(\sigma^{4} \sigma^{\prime 3}\right)^{*}$ and $\bar{L} \tilde{H} S_{R} \sigma^{2} \sigma^{\prime 3}$.

\subsubsection{Model II}

In the second model featuring two ALPs, we require the cancellation of all anomalies, including gravitational anomalies, but we relax the conditions for stability of the ISS mechanism. Because of the former, we can only find a symmetry $\mathbb{Z}_{8} \times \mathbb{Z}_{10}$, so that the ALP $a^{\prime}$ is heavier than the previous model and can account for the $\gamma$-ray transparency problem but not the soft X-ray from the Coma cluster.

The model Lagrangian involving $\sigma, \sigma^{\prime}$ is

$$
\begin{aligned}
-\mathscr{L} \supset & \eta_{i j} \frac{\sigma^{2}}{M_{\mathrm{Pl}}} \overline{S_{R i}} N_{R j}^{c}+\zeta_{i j} \frac{\sigma^{* 2} \sigma^{* *}}{M_{\mathrm{Pl}}^{2}} \overline{S_{R i}} S_{R j}^{c} \\
& +k_{i} \frac{\sigma \sigma^{\prime}}{M_{\mathrm{Pl}}^{2}} \overline{L_{i}} H E_{R}+k_{E} \frac{\sigma \sigma^{\prime}}{M_{\mathrm{Pl}}} \overline{E_{L}} E_{R}+\text { H.c. }
\end{aligned}
$$

The Lagrangian has the form in eq. (2.40) with $(p, q)=(2,-2)$ and $\left(p^{\prime}, q^{\prime}\right)=(0,-1)$, whereas $(r, s)=\left(r^{\prime}, s^{\prime}\right)=(1,1)$. As in the previous model, we choose $v_{\sigma} \approx 2.44 \times 10^{10} \mathrm{GeV}$ and $\sigma$ is protected by $\mathbb{Z}_{8}$. The symmetry $\mathbb{Z}_{10}$ then protects $\sigma^{\prime}$.

After performing the calculations of section 2.1 for this case, we choose $Z=3 \mathcal{L}-3 \mathcal{B}$ and $Z^{\prime}=\mathcal{L}^{\prime}+9 \mathcal{B}$ as generators of $\mathbb{Z}_{8}$ and $\mathbb{Z}_{10}$, respectively; they are given in table 7 . One can check that these charges are anomaly free. The anomalous symmetries $\mathrm{U}(1)_{X}$ and $\mathrm{U}(1)_{X^{\prime}}$ 


\begin{tabular}{|c|c|c|c|c|c|c|c|c|c|c|c|c|}
\hline & $q_{i L}$ & $d_{i R}$ & $u_{i R}$ & $H$ & $L_{i}$ & $l_{i R}$ & $N_{i R}$ & $S_{i R}$ & $E_{L}$ & $E_{R}$ & $\sigma$ & $\sigma^{\prime}$ \\
\hline $\mathbb{Z}_{8}$ & -1 & -1 & -1 & 0 & 3 & 3 & 3 & -1 & 3 & 2 & 1 & 0 \\
\hline $\mathbb{Z}_{10}$ & 3 & 3 & 3 & 0 & 1 & 1 & 1 & -1 & 1 & -1 & 0 & 2 \\
\hline
\end{tabular}

Table 7. $\mathbb{Z}_{8} \times \mathbb{Z}_{10}$ charges in the notation in eq. (2.25).

can be obtained from the same table by eliminating the baryon number contributions and rescaling the $X^{\prime}$ charge of $\sigma^{\prime}$ to unity. The extended lepton numbers $\mathcal{L}$ and $\mathcal{L}^{\prime}$ can be extracted in an analogous manner. They give $C_{a \gamma}=C_{a^{\prime} \gamma}=2$. The ALP-photon couplings are the same as for model I,

$$
g_{a \gamma} \approx 9.52 \times 10^{-14} \mathrm{GeV}^{-1}, \quad g_{a^{\prime} \gamma} \approx 2.32 \times 10^{-12} \mathrm{GeV}^{-1},
$$

for $v_{\sigma} \approx 2.44 \times 10^{10} \mathrm{GeV}$ and $v_{\sigma^{\prime}} \approx 10^{9} \mathrm{GeV}$. The ALP masses are given by

$$
m_{a} \approx|g| \times 7.1 \mathrm{keV}, \quad m_{a^{\prime}} \approx\left|g^{\prime}\right| \times 1.81 \times 10^{-10} \mathrm{eV} .
$$

We can see $m_{a^{\prime}}$ is too large to explain the soft X-ray excess in the Coma cluster. Benchmark ALP photon couplings and masses can seen on figure 2 marked as C.1 and C.2.

Finally, the neutrino mass matrices have magnitude

$$
M=\eta \times 124 \mathrm{GeV}, \quad \mu=\zeta \times 36.5 \mathrm{eV},
$$

which leads to the light neutrino mass matrix

$$
m_{\nu}=\left[y^{\top} \eta^{-1} \zeta\left(\eta^{\top}\right)^{-1} y\right] \times 72 \mathrm{eV} .
$$

In this case, we have a more natural scale for $\mu$ compared to the model of section 2.3.1 but the general considerations for $\eta$ are the same. Concerning the stability of the ISS mechanism, we can see the lowest order operators that disrupt the texture in eq. (2.2) are $N_{R}^{2} \sigma^{2} \sigma^{*}$ and $\bar{L} \tilde{H} S_{R} \sigma^{* 2} \sigma^{\prime}$. They lead respectively to mass parameters of magnitude

$$
\mu_{N} \sim \frac{v_{\sigma}^{2} v_{\sigma^{\prime}}}{2^{3 / 2} M_{\mathrm{Pl}}^{2}} \approx 36.5 \mathrm{eV}, \quad m_{D S} \sim \frac{v_{\mathrm{w}} v_{\sigma}^{2} v_{\sigma^{\prime}}}{2^{4 / 2} M_{\mathrm{Pl}}^{3}} \approx 2.6 \times 10^{-15} \mathrm{eV}
$$

These mass matrices contribute to entries $(3,1)$ and $(2,2)$ in eq. $(2.2)$ and contribute to subleading terms in the light neutrino mass matrix in eq. $(2.3)$ as $[62,63,87]$

$$
\begin{aligned}
\delta m_{\nu}= & -\left[m_{D}^{\top} M^{-1} m_{D S}+m_{D S}^{\top} M^{\top-1} m_{D}\right]-m_{D S}^{\top} M^{\top-1} \mu_{N} M^{-1} m_{D S} \\
& +m_{D}^{\top} M^{-1} \mu M^{\top-1} \mu_{N} M^{-1} m_{D S}+m_{D S}^{\top} M^{\top-1} \mu_{N} M^{-1} \mu M^{\top-1} m_{D} \\
& -m_{D}^{\top} M^{-1} \mu M^{\top-1} \mu_{N} M^{-1} \mu M^{\top-1} m_{D}+O\left(M^{-5}, \mu^{3}, \mu_{N}^{2}\right) .
\end{aligned}
$$

We can see the contribution of $m_{D S}$ is negligible. The mass parameter $\mu_{N}$ is of the order of $\mu$ but it also contributes negligibly to the light neutrino mass matrix, even if one-loop corrections are taken into account [87]. 


\section{Conclusions}

We have generically studied the construction of models where one ALP results from a scalar singlet carrying an anomalous extension of the lepton number $\mathcal{L}$ of the SM. The ALP successfully accounts for some intriguing astrophysical phenomena such as the soft Xray excess in the Coma cluster, and at the same time, the singlet vev furnishes the correct mass scales that implements the ISS mechanism for neutrino mass generation through gravity induced nonrenormalizable terms. Moreover, the approximate nature of anomalous $\mathcal{L}$-number, the ALP mass and the ISS mechanism are protected from additional gravity induced terms through a gauge discrete symmetry. The additional beyond SM fields are minimal: three families of right-handed neutrino fields $N_{i R}, S_{i R}$, one heavy singlet lepton $E$ and one singlet scalar $\sigma$ are added.

By requiring the stability of the ISS mechanism and the cancellation of the discrete gauge anomalies, only one model survives, and the discrete symmetry needs to be a $\mathbb{Z}_{13}$ subgroup of a combination of $\mathcal{L}$ and the baryon number $B$, the simplest being $6 \mathcal{B}+7 \mathcal{L}$.

Simple extensions to models with two ALPs can be constructed by adding solely one more singlet scalar. In this case, two ALPs can solve more astrophysical phenomena with distinct features. We have been unable to find a model capable of explaining the three astrophysical phenomena and, at the same time, satisfying all the conditions in section 2.1. Therefore, two models are presented by relaxing some of the conditions. The first model can explain all the astrophysical phenomena, but the gravitational anomaly for one $\mathbb{Z}_{N}$ factor can not be canceled within the field content, and additional fermionic fields are required. The second model does not present discrete anomaly but it can not explain the soft X-ray excess in the Coma cluster. In both cases, the $7.1 \mathrm{keV}$ ALP may be an appreciable component of dark matter as well [26, 90-92].

In summary, we have proposed a very restrictive and economical setting to extend the SM and explain notable astrophysical phenomena together with natural neutrino mass generation through the ISS mechanism. The restrictiveness of the setting allows only one model with one ALP and models with two ALPs are also largely restricted. More possibilities emerge if we allow the presence of more than one heavy charged lepton and, in particular, three copies of them can lead to easier cancellation of the gravitational anomaly.

\section{Acknowledgments}

The authors thank Andreas Ringwald for very helpful suggestions. This research was supported by Conselho Nacional de Desenvolvimento Científico e Tecnológico (CNPq) (A.G.D and C.D.R.C), and by the grants 2013/22079-8 (A.G.D and C.C.N) and 2013/26371-5 (C.C.N) of Fundação de Amparo à Pesquisa do Estado de São Paulo (FAPESP). C.C.N. also thanks the Maryland Center for Fundamental Physics for its hospitality. B.L.S.V thanks Coordenação Aperfeiçoamento de Pessoal de Nível Superior (CAPES), for the financial support under Contract No. 2264-13-7 and the Argonne National Laboratory for its hospitality. 
Open Access. This article is distributed under the terms of the Creative Commons Attribution License (CC-BY 4.0), which permits any use, distribution and reproduction in any medium, provided the original author(s) and source are credited.

\section{References}

[1] R.D. Peccei and H.R. Quinn, CP Conservation in the Presence of Instantons, Phys. Rev. Lett. 38 (1977) 1440 [INSPIRE].

[2] S. Weinberg, A New Light Boson?, Phys. Rev. Lett. 40 (1978) 223 [INSPIRE].

[3] F. Wilczek, Problem of Strong $p$ and $t$ Invariance in the Presence of Instantons, Phys. Rev. Lett. 40 (1978) 279 [INSPIRE].

[4] J. Jaeckel and A. Ringwald, The Low-Energy Frontier of Particle Physics, Ann. Rev. Nucl. Part. Sci. 60 (2010) 405 [arXiv:1002.0329] [INSPIRE].

[5] A. Ringwald, Exploring the Role of Axions and Other WISPs in the Dark Universe, Phys. Dark Univ. 1 (2012) 116 [arXiv: 1210.5081] [INSPIRE].

[6] R. Essig et al., Working Group Report: New Light Weakly Coupled Particles, arXiv: 1311.0029 [INSPIRE].

[7] R.N. Mohapatra, Mechanism for Understanding Small Neutrino Mass in Superstring Theories, Phys. Rev. Lett. 56 (1986) 561 [InSPIRE].

[8] R.N. Mohapatra and J.W.F. Valle, Neutrino Mass and Baryon Number Nonconservation in Superstring Models, Phys. Rev. D 34 (1986) 1642 [inSPIRE].

[9] L.M. Krauss and F. Wilczek, Discrete Gauge Symmetry in Continuum Theories, Phys. Rev. Lett. 62 (1989) 1221 [INSPIRE].

[10] L.E. Ibáñez and G.G. Ross, Discrete gauge symmetry anomalies, Phys. Lett. B 260 (1991) 291 [INSPIRE].

[11] T. Banks and M. Dine, Note on discrete gauge anomalies, Phys. Rev. D 45 (1992) 1424 [hep-th/9109045] [INSPIRE].

[12] L.E. Ibáñez, More about discrete gauge anomalies, Nucl. Phys. B 398 (1993) 301 [hep-ph/9210211] [INSPIRE].

[13] P. Ramond, Mass hierarchies from anomalies: A peek behind the Planck curtain, hep-ph/9604251 [INSPIRE].

[14] C. Csáki and H. Murayama, Discrete anomaly matching, Nucl. Phys. B 515 (1998) 114 [hep-th/9710105] [INSPIRE].

[15] T. Araki, T. Kobayashi, J. Kubo, S. Ramos-Sanchez, M. Ratz and P.K.S. Vaudrevange, (Non-)Abelian discrete anomalies, Nucl. Phys. B 805 (2008) 124 [arXiv:0805.0207] [INSPIRE].

[16] C. Luhn and P. Ramond, Anomaly Conditions for Non-Abelian Finite Family Symmetries, JHEP 07 (2008) 085 [arXiv:0805.1736] [INSPIRE].

[17] H.K. Dreiner, C. Luhn and M. Thormeier, What is the discrete gauge symmetry of the MSSM?, Phys. Rev. D 73 (2006) 075007 [hep-ph/0512163] [INSPIRE].

[18] G. Lazarides, C. Panagiotakopoulos and Q. Shafi, Phenomenology and Cosmology With Superstrings, Phys. Rev. Lett. 56 (1986) 432 [INSPIRE]. 
[19] M. Dine, Problems of naturalness: Some lessons from string theory, hep-th/9207045 [INSPIRE].

[20] A.G. Dias, V. Pleitez and M.D. Tonasse, Naturally light invisible axion and local $Z_{13} \otimes Z_{3}$ symmetries, Phys. Rev. D 69 (2004) 015007 [hep-ph/0210172] [INSPIRE].

[21] A.G. Dias, V. Pleitez and M.D. Tonasse, Naturally light invisible axion in models with large local discrete symmetries, Phys. Rev. D 67 (2003) 095008 [hep-ph/0211107] [INSPIRE].

[22] K.S. Babu, I. Gogoladze and K. Wang, Stabilizing the axion by discrete gauge symmetries, Phys. Lett. B 560 (2003) 214 [hep-ph/0212339] [INSPIRE].

[23] L.M. Carpenter, M. Dine and G. Festuccia, Dynamics of the Peccei Quinn Scale, Phys. Rev. D 80 (2009) 125017 [arXiv:0906.1273] [INSPIRE].

[24] J.C. Montero and B.L. Sánchez-Vega, Natural PQ symmetry in the 3-3-1 model with a minimal scalar sector, Phys. Rev. D 84 (2011) 055019 [arXiv:1102.5374] [INSPIRE].

[25] K. Harigaya, M. Ibe, K. Schmitz and T.T. Yanagida, Peccei-Quinn symmetry from a gauged discrete $R$ symmetry, Phys. Rev. D 88 (2013) 075022 [arXiv:1308.1227] [InSPIRE].

[26] A.G. Dias, A.C.B. Machado, C.C. Nishi, A. Ringwald and P. Vaudrevange, The Quest for an Intermediate-Scale Accidental Axion and Further ALPs, JHEP 06 (2014) 037 [arXiv: 1403.5760] [INSPIRE].

[27] A. Celis, J. Fuentes-Martín and H. Serôdio, A class of invisible axion models with FCNCs at tree level, JHEP 12 (2014) 167 [arXiv:1410.6218] [INSPIRE].

[28] A. Mirizzi, G.G. Raffelt and P.D. Serpico, Signatures of axion-like particles in the spectra of TeV gamma-ray sources, Phys. Rev. D 76 (2007) 023001 [arXiv:0704.3044] [InSPIRE].

[29] A. De Angelis, M. Roncadelli and O. Mansutti, Evidence for a new light spin-zero boson from cosmological gamma-ray propagation?, Phys. Rev. D 76 (2007) 121301 [arXiv:0707.4312] [INSPIRE].

[30] HESS collaboration, F. Aharonian et al., New constraints on the Mid-IR EBL from the HESS discovery of VHE gamma rays from 1ES 0229+200, Astron. Astrophys. 475 (2007) L9 [arXiv: 0709.4584] [INSPIRE].

[31] MAGIC collaboration, E. Aliu et al., Very-High-Energy Gamma Rays from a Distant Quasar: How Transparent Is the Universe?, Science 320 (2008) 1752 [arXiv:0807.2822] [INSPIRE].

[32] W. Essey and A. Kusenko, On weak redshift dependence of gamma-ray spectra of distant blazars, Astrophys. J. 751 (2012) L11 [arXiv:1111.0815] [INSPIRE].

[33] D. Horns and M. Meyer, Indications for a pair-production anomaly from the propagation of VHE gamma-rays, JCAP 02 (2012) 033 [arXiv:1201.4711] [INSPIRE].

[34] M. Simet, D. Hooper and P.D. Serpico, The Milky Way as a Kiloparsec-Scale Axionscope, Phys. Rev. D 77 (2008) 063001 [arXiv:0712.2825] [INSPIRE].

[35] M.A. Sanchez-Conde, D. Paneque, E. Bloom, F. Prada and A. Dominguez, Hints of the existence of Axion-Like-Particles from the gamma-ray spectra of cosmological sources, Phys. Rev. D 79 (2009) 123511 [arXiv:0905.3270] [INSPIRE].

[36] M. Meyer, D. Horns and M. Raue, First lower limits on the photon-axion-like particle coupling from very high energy gamma-ray observations, Phys. Rev. D 87 (2013) 035027 [arXiv: 1302.1208] [INSPIRE]. 
[37] G.I. Rubtsov and S.V. Troitsky, Breaks in gamma-ray spectra of distant blazars and transparency of the Universe, JETP Lett. 100 (2014) 397 [arXiv:1406.0239] [INSPIRE].

[38] R. Lieu et al., Diffuse Extreme-Ultraviolet Emission from the Coma Cluster: Evidence for Rapidly Cooling Gases at Submegakelvin Temperatures, Science 274 (1996) 1335.

[39] M. Cicoli, J.P. Conlon and F. Quevedo, Dark radiation in LARGE volume models, Phys. Rev. D 87 (2013) 043520 [arXiv: 1208.3562] [INSPIRE].

[40] T. Higaki and F. Takahashi, Dark Radiation and Dark Matter in Large Volume Compactifications, JHEP 11 (2012) 125 [arXiv:1208.3563] [INSPIRE].

[41] J.P. Conlon and M.C.D. Marsh, Excess Astrophysical Photons from a 0.1-1 keV Cosmic Axion Background, Phys. Rev. Lett. 111 (2013) 151301 [arXiv:1305.3603] [INSPIRE].

[42] S. Angus, J.P. Conlon, M.C.D. Marsh, A.J. Powell and L.T. Witkowski, Soft X-ray Excess in the Coma Cluster from a Cosmic Axion Background, JCAP 09 (2014) 026 [arXiv: 1312.3947] [INSPIRE].

[43] E. Bulbul, M. Markevitch, A. Foster, R.K. Smith, M. Loewenstein and S.W. Randall, Detection of An Unidentified Emission Line in the Stacked X-ray spectrum of Galaxy Clusters, Astrophys. J. 789 (2014) 13 [arXiv:1402.2301] [INSPIRE].

[44] A. Boyarsky, O. Ruchayskiy, D. Iakubovskyi and J. Franse, Unidentified Line in X-Ray Spectra of the Andromeda Galaxy and Perseus Galaxy Cluster, Phys. Rev. Lett. 113 (2014) 251301 [arXiv: 1402 .4119] [INSPIRE].

[45] T. Higaki, K.S. Jeong and F. Takahashi, The $7 \mathrm{keV}$ axion dark matter and the X-ray line signal, Phys. Lett. B 733 (2014) 25 [arXiv:1402.6965] [INSPIRE].

[46] J. Jaeckel, J. Redondo and A. Ringwald, $3.55 \mathrm{keV}$ hint for decaying axionlike particle dark matter, Phys. Rev. D 89 (2014) 103511 [arXiv:1402.7335] [InSPIRE].

[47] J. Biteau and D.A. Williams, The extragalactic background light, the Hubble constant and anomalies: conclusions from 20 years of TeV gamma-ray observations, arXiv:1502.04166 [INSPIRE].

[48] K.S. Babu and R.N. Mohapatra, 7keV Scalar Dark Matter and the Anomalous Galactic X-ray Spectrum, Phys. Rev. D 89 (2014) 115011 [arXiv:1404.2220] [InSPIRE].

[49] K.S. Babu, S. Chakdar and R.N. Mohapatra, Warm Dark Matter in Two Higgs Doublet Models, Phys. Rev. D 91 (2015) 075020 [arXiv:1412.7745] [INSPIRE].

[50] F.S. Queiroz and K. Sinha, The Poker Face of the Majoron Dark Matter Model: LUX to keV Line, Phys. Lett. B 735 (2014) 69 [arXiv:1404.1400] [INSPIRE].

[51] V. Berezinsky and J.W.F. Valle, The KeV majoron as a dark matter particle, Phys. Lett. B 318 (1993) 360 [hep-ph/9309214] [INSPIRE].

[52] J.P. Conlon and A.J. Powell, A $3.55 \mathrm{keV}$ line from $\mathrm{DM} \rightarrow a \rightarrow \gamma$ : predictions for cool-core and non-cool-core clusters, JCAP 01 (2015) 019 [arXiv: 1406.5518] [INSPIRE].

[53] S. Riemer-Sorensen, Questioning a 3.5 keV dark matter emission line, arXiv:1405.7943 [INSPIRE].

[54] T.E. Jeltema and S. Profumo, Discovery of a $3.5 \mathrm{keV}$ line in the Galactic Center and a Critical Look at the Origin of the Line Across Astronomical Targets, arXiv:1408.1699 [INSPIRE]. 
[55] A. Boyarsky, J. Franse, D. Iakubovskyi and O. Ruchayskiy, Checking the dark matter origin of $3.53 \mathrm{keV}$ line with the Milky Way center, arXiv:1408.2503 [INSPIRE].

[56] R. Bähre et al., Any light particle search II - Technical Design Report, 2013 JINST 8 T09001 [arXiv: 1302.5647] [INSPIRE].

[57] E. Armengaud et al., Conceptual Design of the International Axion Observatory (IAXO), 2014 JINST 9 T05002 [arXiv: 1401.3233] [INSPIRE].

[58] A. Kogut et al., The Primordial Inflation Explorer (PIXIE): A Nulling Polarimeter for Cosmic Microwave Background Observations, JCAP 07 (2011) 025 [arXiv:1105.2044] [INSPIRE].

[59] PRISM collaboration, P. André et al., PRISM (Polarized Radiation Imaging and Spectroscopy Mission): An Extended White Paper, JCAP 02 (2014) 006 [arXiv:1310.1554] [INSPIRE].

[60] A. Abada, G. Arcadi and M. Lucente, Dark Matter in the minimal Inverse Seesaw mechanism, arXiv:1406.6556 [INSPIRE].

[61] P.S. Bhupal Dev and A. Pilaftsis, Light and Superlight Sterile Neutrinos in the Minimal Radiative Inverse Seesaw Model, Phys. Rev. D 87 (2013) 053007 [arXiv:1212.3808] [INSPIRE].

[62] H. Hettmansperger, M. Lindner and W. Rodejohann, Phenomenological Consequences of sub-leading Terms in See-Saw Formulas, JHEP 04 (2011) 123 [arXiv:1102.3432] [INSPIRE].

[63] W. Grimus and L. Lavoura, The seesaw mechanism at arbitrary order: Disentangling the small scale from the large scale, JHEP 11 (2000) 042 [hep-ph/0008179] [INSPIRE].

[64] S.M. Boucenna, S. Morisi and J.W.F. Valle, The low-scale approach to neutrino masses, Adv. High Energy Phys. 2014 (2014) 831598 [arXiv: 1404.3751] [INSPIRE].

[65] D.V. Forero, S. Morisi, M. Tortola and J.W.F. Valle, Lepton flavor violation and non-unitary lepton mixing in low-scale type-I seesaw, JHEP 09 (2011) 142 [arXiv:1107.6009] [INSPIRE].

[66] A. Ibarra, E. Molinaro and S.T. Petcov, TeV Scale See-Saw Mechanisms of Neutrino Mass Generation, the Majorana Nature of the Heavy Singlet Neutrinos and $(\beta \beta)_{0 \nu}$-Decay, JHEP 09 (2010) 108 [arXiv: 1007.2378] [INSPIRE].

[67] A. Das and N. Okada, Inverse seesaw neutrino signatures at the LHC and ILC, Phys. Rev. D 88 (2013) 113001 [arXiv: 1207.3734] [INSPIRE].

[68] A. Das, P.S. Bhupal Dev and N. Okada, Direct bounds on electroweak scale pseudo-Dirac neutrinos from $\sqrt{s}=8 \mathrm{TeV}$ LHC data, Phys. Lett. B 735 (2014) 364 [arXiv:1405.0177] [INSPIRE].

[69] A.G. Dias, C.A. de S. Pires and P.S.R. da Silva, How the Inverse See-Saw Mechanism Can Reveal Itself Natural, Canonical and Independent of the Right-Handed Neutrino Mass, Phys. Rev. D 84 (2011) 053011 [arXiv:1107.0739] [INSPIRE].

[70] A.G. Dias, C.A. de S. Pires, P.S. Rodrigues da Silva and A. Sampieri, A Simple Realization of the Inverse Seesaw Mechanism, Phys. Rev. D 86 (2012) 035007 [arXiv:1206.2590] [INSPIRE].

[71] F.F. Freitas, C.A. d.S. Pires and P.S.R. da Silva, Inverse Type II Seesaw Mechanism for Neutrino Masses, arXiv:1408.5878 [INSPIRE].

[72] A. Ringwald, Axions and Axion-Like Particles, arXiv:1407.0546 [INSPIRE]. 
[73] J.E. Kim, Light Pseudoscalars, Particle Physics and Cosmology, Phys. Rept. 150 (1987) 1 [INSPIRE].

[74] A. Friedland, M. Giannotti and M. Wise, Constraining the Axion-Photon Coupling with Massive Stars, Phys. Rev. Lett. 110 (2013) 061101 [arXiv:1210.1271] [INSPIRE].

[75] J.A. Grifols, E. Masso and R. Toldra, Gamma-rays from SN1987A due to pseudoscalar conversion, Phys. Rev. Lett. 77 (1996) 2372 [astro-ph/9606028] [INSPIRE].

[76] J.W. Brockway, E.D. Carlson and G.G. Raffelt, SN1987A gamma-ray limits on the conversion of pseudoscalars, Phys. Lett. B 383 (1996) 439 [astro-ph/9605197] [INSPIRE].

[77] A. Payez, C. Evoli, T. Fischer, M. Giannotti, A. Mirizzi and A. Ringwald, Revisiting the SN1987A gamma-ray limit on ultralight axion-like particles, JCAP 02 (2015) 006 [arXiv: 1410.3747] [INSPIRE].

[78] A. Payez, J.R. Cudell and D. Hutsemekers, New polarimetric constraints on axion-like particles, JCAP 07 (2012) 041 [arXiv: 1204.6187] [INSPIRE].

[79] A. Payez, Constraining ALPs with linear and circular polarisation measurements of quasar light, arXiv:1309.6114 [INSPIRE].

[80] M. Cicoli, J.P. Conlon, M.C.D. Marsh and M. Rummel, $3.55 \mathrm{keV}$ photon line and its morphology from a $3.55 \mathrm{keV}$ axionlike particle line, Phys. Rev. D 90 (2014) 023540 [arXiv: 1403.2370] [INSPIRE].

[81] H.M. Lee, S.C. Park and W.-I. Park, Cluster X-ray line at $3.5 \mathrm{keV}$ from axion-like dark matter, Eur. Phys. J. C 74 (2014) 3062 [arXiv: 1403.0865] [INSPIRE].

[82] M.L. Perl et al., The search for stable, massive, elementary particles, Int. J. Mod. Phys. A 16 (2001) 2137 [hep-ex/0102033] [INSPIRE].

[83] CMS collaboration, Searches for long-lived charged particles in pp collisions at $\sqrt{s}=7$ and $8 \mathrm{TeV}, J H E P \quad 07$ (2013) 122 [arXiv:1305.0491] [INSPIRE].

[84] R.N. Mohapatra, From Old Symmetries to New Symmetries: Quarks, Leptons and B-L, Int. J. Mod. Phys. A 29 (2014) 1430066 [arXiv:1409.7557] [inSPIRE].

[85] I.P. Ivanov and C.C. Nishi, Abelian symmetries of the N-Higgs-doublet model with Yukawa interactions, JHEP 11 (2013) 069 [arXiv:1309.3682] [INSPIRE].

[86] C.C. Nishi, Compatible abelian symmetries in N-Higgs-Doublet Models, JHEP 03 (2015) 034 [arXiv: 1411.4909] [INSPIRE].

[87] P.S.B. Dev and A. Pilaftsis, Minimal Radiative Neutrino Mass Mechanism for Inverse Seesaw Models, Phys. Rev. D 86 (2012) 113001 [arXiv:1209.4051] [INSPIRE].

[88] Z.G. Berezhiani and M.Y. Khlopov, Physical and astrophysical consequences of breaking of the symmetry of families (in Russian), Sov. J. Nucl. Phys. 51 (1990) 935 [Yad. Fiz. 51 (1990) 1479] [INSPIRE].

[89] Z.G. Berezhiani and M.Y. Khlopov, The theory of broken gauge symmetry of families (in Russian), Sov. J. Nucl. Phys. 51 (1990) 739 [Yad. Fiz. 51 (1990) 1157] [INSPIRE].

[90] P. Arias, D. Cadamuro, M. Goodsell, J. Jaeckel, J. Redondo and A. Ringwald, WISPy Cold Dark Matter, JCAP 06 (2012) 013 [arXiv:1201.5902] [INSPIRE].

[91] D.J.E. Marsh and J. Silk, A Model For Halo Formation With Axion Mixed Dark Matter, Mon. Not. Roy. Astron. Soc. 437 (2013) 2652 [arXiv:1307.1705] [INSPIRE].

[92] K. Kadota, Y. Mao, K. Ichiki and J. Silk, Cosmologically probing ultra-light particle dark matter using $21 \mathrm{~cm}$ signals, JCAP 06 (2014) 011 [arXiv:1312.1898] [INSPIRE]. 